\title{
TORSION-GROUPS OF ABELIAN COVERINGS OF LINKS
}

BY

\author{
JOHN P. MAYBERRY AND KUNIO MURASUGI
}

\begin{abstract}
If $M$ is an abelian branched covering of $S^{3}$ along a link $L$, the order of $H_{1}(M)$ can be expressed in terms of (i) the Alexander polynomials of $L$ and of its sublinks, and (ii) a "redundancy" function characteristic of the monodromy-group. In 1954, the first author thus generalized a result of Fox (for $L$ a knot, in which case the monodromy-group is cyclic and the redundancy trivial); we now prove earlier conjectures and give a simple interpretation of the redundancy. Cyclic coverings of links are discussed as simple special cases.

We also prove that the Poincare conjecture is valid for the above-specified family of 3-manifolds $M$.

We state related results for unbranched coverings.
\end{abstract}

0. Introduction. If $K$ is a knot in $S^{3}$, any abelian representation of $\pi_{1}\left(S^{3}-K\right)$ must be cyclic because $H_{1}\left(S^{3}-K ; \mathbf{Z}\right)$ is infinite cyclic. The associated branched covering space $M$ and unbranched covering space $U$ are called cyclic coverings of the knot $K$; such coverings have been studied extensively (for example, in $[1,7,20]$ and [22]). In particular, a classical result of Fox [7, p. 417] gives the order of the first homology group of $M$ easily in terms of the Alexander polynomial of $K$. (Closely related results had been obtained earlier by Zariski; his paper [24] and Goeritz' [13a] were focused on the Betti number of $M$.)

The Alexander polynomial of a link, as defined by Fox [6], is not an immediate generalization of that of a knot. Consequently, complications often arise in generalizing (to links) results which involve the Alexander polynomials of knots. Our main result, Theorem 10.1, generalizes the above result of Fox; both the result, and our methods, involve complications far beyond those needed in the classical case. (We need, for example, to develop algebraic relationships between the eigenvalues of an integral matrix and its elementary divisors.)

In 1955, one author of this paper announced [19] a formula for the order of the first homology group of an arbitrary abelian covering of a link $L$ (i.e., a regular covering with abelian monodromy-group). The importance of abelian coverings is due to two facts. First, their existence is easy to decide; every transitive abelian permutation group $\mathfrak{N}$ with a $\mu$-element generating set $\left(m_{1}, m_{2}, \ldots, m_{\mu}\right)$ is the monodromy-group of a unique covering $C$ for any link $L$ of $\mu$ components. Second,

Received by the editors October 2, 1979 and, in revised form, December 3, 1980.

1980 Mathematics Subject Classification. Primary 57M25; Secondary 57M12, 57M40, 20 K01.

Key words and phrases. Knots, links, unbranched coverings, branched coverings, abelian coverings, monodromy-groups, Seifert matrices, elementary divisors, Alexander matrix, Alexander polynomial, Poincaré conjecture, homology spheres, redundancy of a covering, 3-manifolds, cyclic covering. 
given such a group $\Re$ and two $\mu$-component links $L_{1}$ and $L_{2}$, a natural correspondence between the respective coverings $C_{1}$ and $C_{2}$ can be established, which must be a homeomorphism if $L_{1}$ and $L_{2}$ are equivalent.

In [19] it was proved that the order of the first homology group of an abelian covering is not determined by the Alexander polynomial of $L$, but depends also on (i) the Alexander polynomials of sublinks of $L$, and (ii) a numerical function $D(\omega)$ of the covering. $D(\omega)$, which we now call the "redundancy" of the covering, was given by an explicit but very complicated expression; actual values of $D(\omega)$ were found only for a few very special cases (including the cyclic coverings, and all coverings of 2-component links), though values for some other cases were conjectured.

Hosokawa and Kinoshita later showed [17] that the order $T_{n}(L)$ of the first homology group of a cyclic ${ }^{1} n$-sheeted covering space of the link $L$ depends only on $n$ and a certain polynomial which is entirely determined by the Alexander polynomial of $L$. Their result was simpler than that of [19], because for the coverings they considered the function $D(\omega)$ is always equal to $n$, and the Alexander polynomials of the sublinks do not occur; it was subsumed in one of the cases previously known explicitly. Their method, though much simpler than that of [19], is not applicable to noncyclic coverings; see $\$ 11$ for examples and further discussion.

One of the purposes of this paper is to prove several explicit and convenient formulae for the redundancy function $D(\omega)$, and to demonstrate the truth of all the conjectures of [19]; it turns out that this "redundancy", which depends only on the structure of the covering and not at all on the link involved, may have some independent value as a tool for studying presentations of finite abelian groups.

Another purpose of this paper is to show certain new restrictions, which must be satisfied by any $\mathbf{Z}$-homology sphere which can occur as an abelian covering of a knot or link. Those restrictions, summarized in Theorem 12.2, can be combined with a recently proven theorem, ${ }^{2}$ "A cyclic branched covering of a nontrivial knot is never simply-connected", to show (Theorem 13.1) that any simply-connected abelian covering of a link must be $S^{3}$.

However, it is known [22a] that there is a closed orientable 3-manifold which cannot be obtained as an abelian covering (or even as a regular covering) of any link. We note that J. M. Montesinos has shown [19a] that $S^{1} \times S^{1} \times S^{1}$ cannot be a cyclic covering of any knot, but it is easy to see that it is an abelian covering of a link.

In any case, our results have extended the validity of the Poincare conjecture to an enlarged family of 3-manifolds-the abelian branched coverings of links.

This paper consists of two chapters: Chapter I (\$§1-5) presents the purely algebraic results which are our basic tools, while Chapter II (\$§6-14) presents the topological applications of those tools. The essential part of [19] is included in $\S \S 6-10$, culminating in our main Theorem 10.1 , which considerably extends that earlier work. $\$ 11$ discusses the problem of correctly generalizing to links the notion

\footnotetext{
'Called in this paper "strictly-cyclic"- $\$ 11$.

${ }^{2}$ Personal communication from C. McA. Gordon.
} 
of "cyclic covering" of knots, and presents examples which clarify the relationship of our main theorem with the work of Hosokawa and Kinoshita. In $\$ 12$ we explain a classical example (due to Seifert) of a $\mathbf{Z}$-homology sphere from our point of view. In $\$ 13$ we prove the Poincare conjecture for abelian branched coverings of links. Finally, in $\S 14$, we state without proof several related theorems on homology groups of unbranched coverings of links; those theorems imply certain restrictions on the Alexander polynomials of links, and may contribute to the characterization of such polynomials.

Throughout this paper, we work only in the piecewise-linear category, consisting of simplicial complexes and piecewise-linear maps.

The following symbols are used throughout the paper.

$M^{t}$ is the transpose of a matrix $M$.

$I_{n}$ is the identity matrix of rank $n$.

$O_{n, m}$ is the $n \times m$ zero matrix; $O_{*, m}$ is a zero matrix of $m$ columns.

$M_{1} \oplus M_{2} \oplus \cdots \oplus M_{n}$ is the block diagonal matrix with diagonal blocks $M_{1}, M_{2}, \ldots, M_{n}$.

$\operatorname{diag}\left\{a_{1}, \ldots, a_{n}\right\}$ is the $n \times n$ matrix with entries $a_{1}, a_{2}, \ldots, a_{n}$ on the principal diagonal, zeros elsewhere.

$J_{n}$ is the set of $n$ elements $\{1,2, \ldots, n\}$.

$\delta\left(J_{n}\right)$ is the symmetric group of permutations on $J_{n}$.

$\mathrm{GL}(n, R)$ is the general linear group of degree $n$ over a commutative ring $R$.

$\square$ indicates the end of a lemma, theorem, proposition, or corollary.

For $g_{i}$ in a group $G,\left\langle g_{1}, \ldots, g_{m}\right\rangle$ is the subgroup of $G$ generated by $g_{1}, \ldots, g_{m}$.

For a subgroup $H$ of $G,|H|$ is the order of $H$.

For $g \in G,|g|=|\langle g\rangle|$.

$C_{\text {is }}$ the field of complex numbers.

$A_{\mu}$ is the free abelian group of rank $\mu$; we take its generators to be $t_{1}, t_{2}, \ldots, t_{\mu}$.

$\mathbf{Z}_{n}$ is the cyclic group of order $n$.

$\mathbf{Z}$ is the ring of integers.

$S^{3}$ is the 3-sphere.

$G(L)$ is the fundamental group of the complement $S^{3}-L$ of the link $L$.

$\alpha \beta x$ or $x^{\alpha \beta}$ may be used for $\alpha(\beta(x))$ or $\left(x^{\beta}\right)^{\alpha}$, where $\alpha$ and $\beta$ are maps.

\section{CHAPTER I}

1. The terminant of a matrix. The elementary divisors $e_{1}, e_{2}, \ldots, e_{n}$ of an $n \times n$ integral matrix $M$ are related to the eigenvalues $\lambda_{1}, \lambda_{2}, \ldots, \lambda_{n}$ of $M$ in an obvious way, since

$$
\prod_{i=1}^{n} e_{i}=\operatorname{det}(M)=\prod_{i=1}^{n} \lambda_{i}
$$

However, if $M$ is singular, both products vanish and the above relation gives no new information.

In case we know a matrix $R$, whose rows happen to express all the dependencies among the rows of $M$, and whose rows also express the dependencies among the 
columns of $M$, we can find a deeper relation between the nonzero elementary divisors and the nonzero eigenvalues, using the numerical function of $R$ which we call the terminant.

Let $\theta$ be a principal ideal domain. Let $R$ be an $n \times n$ matrix over $\theta$.

Definition 1.1. The terminant $F(R)$ is defined as the product of the nonzero elementary divisors of the symmetric matrix $R R^{t}$ over $\theta$.

We ask: Under what conditions will the terminants of two matrices be equal? We note that $F(R)$ is not an invariant under the usual equivalence relation, as is shown by the following example.

EXAMPLE. Let $R_{1}=(1,2)$ and $R_{2}=(1,0)$ be matrices over $\mathbf{Z}$. Then $F\left(R_{1}\right)=5$ but $F\left(R_{2}\right)=1$. On the other hand, $F\left(R_{1}^{t}\right)=F\left(R_{2}^{t}\right)=1$.

We define the relation of $r$-equivalence as follows.

Definition 1.2. Two matrices, $A, B$, over $\theta$ are said to be $r$-equivalent, $A \sim_{r} B$, if there exists a sequence $A=A_{0}, A_{1}, A_{2}, \ldots, A_{k}=B$ such that $A_{i+1}$ is obtained from $A_{i}$ by one of the following operations or their inverses.

(a) $A \rightarrow V A$ with an invertible matrix $V$,

(b) $A \rightarrow A U$ with an orthogonal matrix $U$,

(c) delete a zero column from $A$,

(d) delete a zero row of $A$.

Obviously, $\sim_{r}$ is an equivalence relation.

Remark 1.2.1. By using (a) and (d), one can add or delete a row that is a linear combination of other rows of $A$.

It is easy to see that the terminant is invariant under $\sim_{r}$. That is,

Proposition 1.1. If $A \sim_{r} B$, then $F(A)=F(B)$.

Now, if a matrix $R$ contains an invertible minor of the highest rank, then we can give an alternate expression for $F(R)$ as follows.

Proposition 1.2. Let $R$ be an $n \times m$ matrix over $\theta$ of ranks. Suppose that $R$ contains an invertible $s \times$ s minor $P_{1}$ over $\Theta$. Write

$$
U R V=\left[\begin{array}{ll}
P_{1} & P_{2} \\
P_{3} & P_{4}
\end{array}\right]
$$

with permutation matrices $U, V$. Define $W=P_{1}^{-1} P_{2}$. Then $F(R)=\operatorname{det}\left(I_{s}+W W^{t}\right)$.

Proof. Denote by $Y$ the $s \times m$ matrix $\left[I_{s} W\right] \cdot \operatorname{rank}(Y)=s$, so $Y Y^{t}$ is nonsingular. Then

$$
\begin{aligned}
R & \sim_{r} U R V \\
& \sim_{r}\left[P_{1} P_{2}\right] \text { by Remark 1.2.1, } \\
& \sim_{r} P_{1}^{-1}\left[P_{1} P_{2}\right]=Y,
\end{aligned}
$$

so $F(R)=F(Y)=\operatorname{det}\left(Y Y^{t}\right)=\operatorname{det}\left(I_{s}+W W^{t}\right)$.

2. Eigenvalues and elementary divisors. Now we use the concept of the terminant to prove Theorem 2.1, which provides the first of the promised relationships between the eigenvalues and the elementary divisors of a possibly singular matrix $M$. 
It is important, for our subsequent use of these results, to note that the ratio $\left(\Pi \lambda_{i}\right) /\left(\Pi e_{i}\right)$ is determined solely by the matrix $R$ of dependencies of $M$.

LeMMA 2.1. Let $X$ be an $n \times m$ matrix over the reals, and $Y$ an $m \times n$ matrix. Then

$$
\operatorname{det}\left(I_{n}+X Y\right)=\operatorname{det}\left(I_{m}+Y X\right) \text {. }
$$

Proof. Both determinants retain the same value if we extend $X$ and $Y$ with rows or columns of zeros to produce square matrices $X^{\prime}, Y^{\prime}$; thus it suffices to prove the lemma for square matrices. Since the determinant is a continuous function, we may assume $X, Y$ nonsingular. But then

$$
\operatorname{det}(I+X Y)=\operatorname{det}\left(X^{-1}(I+X Y) X\right)=\operatorname{det}(I+Y X) .
$$

COROLlaRY 2.1.1. For any real $n \times m$ matrix $W$,

$$
\operatorname{det}\left(I_{n}+W W^{t}\right)=\operatorname{det}\left(I_{m}+W^{t} W\right) .
$$

THEOREM 2.1. Let $R$ be an $n \times m$ integer matrix of rank $s$ with $s \times s$ invertible minor $P_{1}$ and terminant $F(R)$. Suppose there is an $m \times m$ integer matrix $M$ satisfying

$$
R M=0 \text { and } M R^{t}=0 .
$$

Let $\lambda_{1}, \lambda_{2}, \ldots, \lambda_{m}$ be the eigenvalues of $M$ with $\left|\lambda_{1}\right| \geqslant\left|\lambda_{2}\right| \geqslant \cdots \geqslant\left|\lambda_{m}\right|$ and $e_{1}, e_{2}, \ldots, e_{m}$ the elementary divisors of $M$ with $e_{i} \mid e_{i+1}, i=1, \ldots, m-1$. (We allow that $0 \mid 0$.) Then,

(a) at least $s$ eigenvalues of $M$ are 0 , i.e. $\lambda_{m-s+1}=\cdots=\lambda_{m}=0$;

(b) at least s elementary divisors of $M$ are 0 , i.e. $e_{m-s+1}=\cdots=e_{m}=0$;

(c) $\prod_{i=1}^{m-s} \lambda_{i}=F(R) \prod_{i=1}^{m-s} e_{i}$;

and (hence) if rank $M=m-s$, then $\lambda_{i} \neq 0$ for $i=1, \ldots, m-s$.

Proof. Write

$$
U R V=\left[\begin{array}{ll}
P_{1} & P_{2} \\
P_{3} & P_{4}
\end{array}\right],
$$

with permutation matrices $U, V$. As in Proposition 1.2, we see $F(R)=F\left(\left[P_{1} P_{2}\right]\right)$.

If $M$ satisfies (2.1), then $\left[\begin{array}{ll}P_{1} & P_{2}\end{array}\right] M^{\prime}=0$ and $M^{\prime}\left[P_{1} P_{2}\right]^{t}=0$, where $M^{\prime}=V^{t} M V$, so $M^{\prime}$ has the same elementary divisors as $M$ and the same eigenvalues as $M$. Therefore, we may assume without loss of generality that $R=\left[\begin{array}{ll}P_{1} & P_{2}\end{array}\right]$ and that $n=s$.

Now write

$$
M=\left[\begin{array}{ll}
M_{1} & M_{2} \\
M_{3} & M_{4}
\end{array}\right],
$$

where $M_{1}$ is an $s \times s$ matrix, and let

$$
X=\left[\begin{array}{cc}
I_{s} & W \\
0 & I_{m-s}
\end{array}\right],
$$


where $W=P_{1}^{-1} P_{2}$. Then $R M=0$ and $M R^{t}=0$ show

$$
\begin{gathered}
P_{1}^{-1} R M=\left[M_{1}+W M_{3}, M_{2}+W M_{4}\right]=0, \\
M R^{t} P_{1}^{-t}=\left[\begin{array}{c}
M_{1}+M_{2} W^{t} \\
M_{3}+M_{4} W^{t}
\end{array}\right]=0 .
\end{gathered}
$$

Further,

$$
X M X^{t}=\left[\begin{array}{cc}
0 & 0 \\
0 & M_{4}
\end{array}\right]
$$

and

$$
\begin{aligned}
X M X^{-1} & =\left(X M X^{t}\right)\left(X X^{t}\right)^{-1} \\
& =\left[\begin{array}{cc}
0 & 0 \\
-M_{4} W^{t} & M_{4}\left(I+W^{t} W\right)
\end{array}\right]
\end{aligned}
$$

so $M$ has at least $s$ zero eigenvalues, proving (a).

Also, $\prod_{i=1}^{m-s} \lambda_{i}=\operatorname{det}\left(I+W^{t} W\right) \cdot \operatorname{det} M_{4}$. Obviously, $\operatorname{det} M_{4}=\prod_{i=1}^{m-s} e_{i}$ and $e_{m-s+1}=\cdots=e_{m}=0$. This proves (b).

If rank $M=m-s$, then $\operatorname{det} M_{4} \neq 0$. Since $\operatorname{det}\left(I+W^{t} W\right) \neq 0$, it follows that $\prod_{i=1}^{m-s} \lambda_{i} \neq 0$. (c) now follows from Proposition 1.2 and Corollary 2.1.1.

3. $r$-equivalence of regular representations. In this section, we define the terminant $F(\omega)$ for any permutation representation $\omega$ of the free abelian group $A_{\mu}$. We show that $F(\omega)$ is an invariant of certain equivalence classes of representations of an abelian group. The definition of $F(\omega)$ is related to the terminant $F(R)$ of a matrix $R$.

Let $\omega: A_{\mu} \rightarrow \delta\left(J_{n}\right)$ be a transitive permutation representation of $A_{\mu}$. That is, $\omega$ is a homomorphism from $A_{\mu}$ into the symmetric group $\varsigma\left(J_{n}\right)$. Since we only consider transitive representations, we simply call $\omega$ a representation. $\omega A_{\mu}$ is denoted by $\mathfrak{R}$, and $\omega t_{i}$ by $m_{i}$ for $i=1,2, \ldots, \mu$.

DEFINITION 3.1. A standard representation matrix will be defined corresponding to each of the generators $t_{1}, t_{2}, \ldots, t_{\mu}$ of $A_{\mu}$. The $i$ th standard representation matrix of $\omega$ is denoted by $B_{i}(\omega)$, or simply by $B_{i}$, and is defined as the $(0,1)$ matrix whose columns correspond to elements of $J_{n}$, with each row the characteristic function of a cycle of $m_{i}$.

Definition 3.2. The terminant $F(\omega)$ of the representation $\omega$ is defined as the terminant of $R(\omega)$, where

$$
R(\omega)=\left[\begin{array}{ccc}
B_{1} & & 0 \\
\vdots & \ddots & \vdots \\
0 & & B_{\mu} \\
I_{n} & \cdots & I_{n}
\end{array}\right]
$$

(see $\S 1)$. I.e., $F(\omega)=F(R(\omega))$.

Definition 3.3. Two representations $\omega_{1}, \omega_{2}: A_{\mu} \rightarrow \delta\left(J_{n}\right)$ are said to be $r$-equivalent, $\omega_{1} \sim_{r} \omega_{2}$, if there exist an inner automorphism $\sigma: \delta\left(J_{n}\right) \rightarrow \delta\left(J_{n}\right)$ and a permutation $\rho$ of the set of generators $\left\{t_{1}, t_{2}, \ldots, t_{\mu}\right\}$ such that $\sigma \omega_{1}=\omega_{2} \rho$. 
Proposition 3.1. If $\omega_{1} \sim_{r} \omega_{2}$, then $F\left(\omega_{1}\right)=F\left(\omega_{2}\right)$.

The proof is straightforward and is omitted.

An element $u$ of $\delta\left(J_{n}\right)$ is called regular if every cycle of the permutation $u$ is of the same length. A representation $\omega: G \rightarrow \delta\left(J_{n}\right)$ is called regular if $\omega(g)$ is regular for each $g \in G$. Since $\omega\left(A_{\mu}\right)$ is abelian and transitive, it is easily seen to be regular.

The rest of $\S \S 3$ and 4 are devoted to deriving an explicit formula for the terminant $F(\omega)$ of a representation $\omega$.

If the standard representation matrix $B_{i}(\omega)$ has $n_{i}$ rows, and $\lambda_{i}$ is the order of $\omega\left(t_{i}\right)$, then $n_{i} \lambda_{i}=n$.

LEMMA 3.1. Let $P_{i}=\tau \omega\left(t_{i}\right)$, where $\tau: \delta\left(J_{n}\right) \rightarrow \mathrm{GL}(n, \mathbf{Z})$ is a regular matrix representation; then

(1) rank $B_{i}=n_{i}$;

(2) $B_{i} B_{i}^{t}=\lambda_{i} I_{n_{i}}$;

(3) $B_{i}^{t} B_{i}=I_{n}+P_{i}+P_{i}^{2}+\cdots+P_{i}^{\lambda_{i}-1}$.

Proof. Immediate from Definition 3.1.

Lemma 3.2. The matrix $R(\omega)$, appearing in Definition 3.2, satisfies

(1) rank $R(\omega)=n_{\omega}+n-1$, and

(2) rank $R(\omega) R(\omega)^{t}=n_{\omega}+n-1$, where $n_{\omega}$ denotes $n_{1}+n_{2}+\cdots+n_{\mu}$.

Proof. Since the sum of the first $n_{\omega}$ rows is equal to the sum of the last $n$ rows, rank $R(\omega) \leqslant n_{\omega}+n-1$; but rank $R(\omega) \geqslant n_{\omega}+n-1$ since $\omega$ is transitive, so $\operatorname{rank} R(\omega)=n_{\omega}+n-1$.

(2) follows from the Binet-Cauchy formula; see for example [18a, p. 38].

Now actual computation shows that

$$
R(\omega) R(\omega)^{t}=\left[\begin{array}{ccccc}
\lambda_{1} I_{n_{1}} & & & & B_{1} \\
& \lambda_{2} I_{n_{2}} & & 0 & B_{2} \\
& & \ddots & & \vdots \\
& 0 & & \lambda_{\mu} I_{n_{\mu}} & B_{\mu} \\
B_{1}^{t} & B_{2}^{t} & \ldots & B_{\mu}^{t} & \mu I_{n}
\end{array}\right] .
$$

Since the sum of the first $n_{\omega}$ rows (and columns) of $R(\omega) R(\omega)^{t}$ is equal to the sum of the last $n$ rows (and columns) and since the rank of $R(\omega) R(\omega)^{t}$ is $n_{\omega}+n-1$, it follows that $F(\omega)$ is given by the value of any principal minor of order $n_{\omega}+n-1$ in $R(\omega) R(\omega)^{t}$, and hence

$$
F(\omega)=\lambda_{1}^{n_{1}} \lambda_{2}^{n_{2}} \cdots \lambda_{\mu}^{n_{\mu}}|\tilde{C}(\omega)|,
$$

where $\tilde{C}(\omega)$ is any principal minor of degree $n-1$ of the matrix

$$
C(\omega)=\mu I_{n}-\sum_{i=1}^{\mu} \frac{1}{\lambda_{i}} B_{i}^{t} B_{i} .
$$


In order to obtain a formula for $F(\omega)$, it only remains to find the eigenvalues of $C(\omega)$.

Let $Z=\left\{\zeta_{1}, \zeta_{2}, \ldots, \zeta_{n}\right\}$ be the set of irreducible representations of $\omega\left(A_{\mu}\right)$ over the field $\Theta$ of complex numbers, with $\zeta_{1}$ the trivial representation.

Let $\tau: \varsigma\left(J_{n}\right) \rightarrow \mathrm{GL}(n, \mathbf{Z})$ be a matrix representation as before.

Since $\omega\left(A_{\mu}\right)$ is abelian, the matrices $P_{i}=\tau \omega\left(t_{i}\right)$ commute; therefore, there exists a matrix $T$ that diagonalizes all $P_{i}$ simultaneously. In fact,

$$
T P_{i} T^{-1}=\operatorname{diag}\left\{\zeta_{1} \omega\left(t_{i}\right), \zeta_{2} \omega\left(t_{i}\right), \ldots, \zeta_{n} \omega\left(t_{i}\right)\right\} .
$$

Now Lemma 3.1(3) implies that

$$
T B_{i}^{t} B_{i} T^{-1}=\operatorname{diag}\left\{\rho_{i, 1}, \rho_{i, 2}, \ldots, \rho_{i, n}\right\},
$$

where $\rho_{i, j}=\sum_{k^{\prime}=0}^{\lambda_{1}-1} \zeta_{j} \omega\left(t_{i}\right)^{k}$.

Since $B_{i}^{t} B_{i}$ and $B_{i} B_{i}^{t}=\lambda_{i} I_{n_{i}}$ have the same nonzero eigenvalues, it follows that $\rho_{t, j}=\lambda_{i}$ if $\zeta_{j} \omega\left(t_{i}\right)=1$ and $\rho_{i, j}=0$ otherwise.

Definition 3.4. $\Lambda\left(\zeta_{j}\right)=\left\{k \mid \zeta_{j} \omega\left(t_{k}\right) \neq 1\right\}$. In particular, $\Lambda\left(\zeta_{j}\right)$ is empty iff $j=1$.

Now (3.3) implies that the eigenvalues of $\sum_{i=1}^{\mu}\left(1 / \lambda_{i}\right) B_{i}^{t} B_{i}$ are exactly

$$
\mu-\left|\Lambda\left(\zeta_{1}\right)\right|, \mu-\left|\Lambda\left(\zeta_{2}\right)\right|, \ldots, \mu-\left|\Lambda\left(\zeta_{n}\right)\right| .
$$

Therefore we have

Proposition 3.2. The eigenvalues of $C(\omega)$ are $0=\left|\Lambda\left(\zeta_{1}\right)\right|,\left|\Lambda\left(\zeta_{2}\right)\right|, \ldots,\left|\Lambda\left(\zeta_{n}\right)\right|$.

Definition 3.5. We define $Q(\omega)=\prod_{i=2}^{n}\left|\Lambda\left(\zeta_{i}\right)\right|$. Note that $Q(\omega) \neq 0$.

Proposition 3.2 now implies

Proposition 3.3. $F(\omega)=Q(\omega) \lambda_{1}^{n_{1}} \lambda_{2}^{n_{2}} \cdots \lambda_{\mu}^{n_{\mu}} / n$.

Proof. Let $p(x)=\sum_{k=0}^{n}(-1)^{k} a_{k} x^{k}$ be the characteristic polynomial of $C(\omega)$. Since any principal minor of degree $n-1$ of $C(\omega)$ has the same value $\tilde{C}(\omega)$, it follows that $a_{1}=n \tilde{C}(\omega)$. On the other hand, $0,\left|\Lambda\left(\zeta_{2}\right)\right|, \ldots,\left|\Lambda\left(\zeta_{n}\right)\right|$ are the eigenvalues of $C(\omega)$ and, hence, $a_{1}=\left|\Lambda\left(\zeta_{2}\right)\right|\left|\Lambda\left(\zeta_{3}\right)\right| \cdots\left|\Lambda\left(\zeta_{n}\right)\right|=Q(\omega)$. Therefore, $\tilde{C}(\omega)=Q(\omega) / n$ and Proposition 3.3 follows immediately.

4. The redundancy $D(\omega)$ of a representation. In this section we define the redundancy $D(\omega)$ of any transitive representation $\omega: A_{\mu} \rightarrow \delta\left(J_{n}\right)$.

Proposition 4.2 shows that $D(\omega)$ is invariant under $r$-equivalence of representations (Definition 3.3 above). It was discovered in [19] that $1 \leqslant D(\omega) \leqslant n$ in some special cases; we prove in Theorem 4.2 that $D(\omega)$ is always an integer between 1 and $n$. The same "redundancy" was chosen because (as shown in Theorem 4.6) values of $D(\omega)$ greater than 1 are associated with representations $\omega$ for which the permutation group $\omega\left(A_{\mu}\right)$ is generated by some proper subset of the generators $\omega\left(t_{1}\right)$, $\omega\left(t_{2}\right), \ldots, \omega\left(t_{\mu}\right)$.

Definition 4.1. Define

$$
\Gamma_{k}=\left\{\zeta \in Z \mid \zeta \omega\left(t_{i}\right) \neq 1 \text { for exactly } k \text { distinct } t_{i}\right\} .
$$

In particular: $\Gamma_{0}=\left\{\zeta_{1}\right\} ; \Gamma_{i} \cap \Gamma_{j}=\varnothing$ if $i \neq j$; and $\zeta \in \Gamma_{k}$ iff $|\Lambda(\zeta)|=k$. 
Definition 4.2. For $k=1,2, \ldots, \mu$, define

$$
U_{k}(\omega)=\prod_{\zeta \in \Gamma_{k}} \prod_{i \in \Lambda(\zeta)}\left(1-\zeta \omega\left(t_{i}\right)\right) .
$$

With these definitions, we can prove

Proposition 4.1. $U_{1}(\omega) U_{2}(\omega) \cdots U_{\mu}(\omega)=\lambda_{1}^{n_{1}} \lambda_{2}^{n_{2}} \cdots \lambda_{\mu}^{n_{\mu}}$.

To prove Proposition 4.1, we need two lemmas concerning the nonnegative integers $a_{1}, a_{2}, \ldots, a_{n}$. We denote by $\left(a_{1}, a_{2}, \ldots, a_{n}\right)$ the greatest common divisor of $a_{1}, a_{2}, \ldots, a_{n}$, and by $\left[a_{1}, a_{2}, \ldots, a_{n}\right]$ their least common multiple.

Then it is easy to prove by induction on $m$ the following lemmas.

LEMMA 4.1 .

$$
\left[\frac{a_{1}}{\left(a_{1}, b\right)}, \frac{a_{2}}{\left(a_{2}, b\right)}, \ldots, \frac{a_{m}}{\left(a_{m}, b\right)}\right] b=\left[a_{1}, a_{2}, \ldots, a_{m}, b\right] .
$$

LemMA 4.2. Let $e_{1}, e_{2}, \ldots, e_{m}$ be positive integers with $e_{1}\left|e_{2}, e_{2}\right| e_{3}, \ldots, e_{m-1} \mid e_{m}$, and set $r_{i}=e_{m} / e_{i}$ for $i=1,2, \ldots, m$. For each $i=1,2, \ldots, m$, let $a_{i}$ be an integer with $0 \leqslant a_{i}<e_{i}$. Then the equation

$$
a_{1} r_{1} X_{1}+a_{2} r_{2} X_{2}+\cdots+a_{m} r_{m} X_{m} \equiv 0 \quad\left(\bmod e_{m}\right)
$$

has $N$ distinct solutions with $0 \leqslant X_{i}<e_{i}$, where

$$
N=\frac{e_{1} e_{2} \cdots e_{m}}{\left[e_{1} /\left(e_{1}, a_{1}\right), e_{2} /\left(e_{2}, a_{2}\right), \ldots, e_{m} /\left(e_{m}, a_{m}\right)\right]} .
$$

Proof of Proposition 4.1. Express the abelian group $\omega\left(A_{\mu}\right)$ as a canonical product of cycles

$$
\omega\left(A_{\mu}\right)=\mathbf{Z}_{e_{1}} \times \mathbf{Z}_{e_{2}} \times \cdots \times \mathbf{Z}_{e_{m}},
$$

where $e_{i} \mid e_{i+1}$ for $1 \leqslant i \leqslant m-1$. Then $n=\left|\omega\left(A_{\mu}\right)\right|=e_{1} e_{2} \cdots e_{m}$. Let $\xi$ be a primitive $e_{m}$ th root of unity. For each $i=1,2, \ldots, m$, choose a generator $u_{i}$ of $\mathbf{Z}_{e}$, and let $r_{i}$ denote $e_{m} / e_{i}$.

Then any irreducible representation $\zeta$ of $\omega\left(A_{\mu}\right)$ must carry each generator $u_{i}$ into some power of the primitive $e_{i}$ th root of unity $\xi^{r_{i}}$; we suppose $\zeta\left(u_{i}\right)=\xi^{r_{1} q_{i}}$. Each such representation $\zeta$ corresponds to a different set $\left\{q_{1}, q_{2}, \ldots, q_{m}\right\}$ with $0 \leqslant q_{i}<e_{i}$, and

$$
\zeta\left(u_{1}^{s_{1}} u_{2}^{s_{2}} \cdots u_{m}^{s_{m}}\right)=\xi^{r_{1} s_{1} q_{1}+\cdots+r_{m} s_{m} q_{m}} .
$$

Letting $\alpha_{i j}$ be defined by the relation

$$
\omega\left(t_{i}\right)=u_{1}^{\alpha_{11}} \cdot u_{2}^{\alpha_{12}} \cdots u_{m}^{\alpha_{i m}},
$$

we see that $\zeta \omega\left(t_{i}\right)=1$ iff

$$
\alpha_{i 1} r_{1} q_{1}+\alpha_{i 2} r_{2} q_{2}+\cdots+\alpha_{i m} r_{m} q_{m} \equiv 0 \quad\left(\bmod e_{m}\right) .
$$

The number of $m$-tuples satisfying (4.2) is given by Lemma 4.2 as

$$
N=\frac{e_{1} \cdot e_{2} \cdots e_{m}}{\left[e_{1} /\left(e_{1}, \alpha_{i 1}\right), e_{2} /\left(e_{2}, \alpha_{i 2}\right), \ldots, e_{m} /\left(e_{m}, \alpha_{i m}\right)\right]} .
$$


Since the $j$ th entry in the denominator of $N$ is simply the order of $u_{j}^{\alpha_{\prime \prime}}$, the denominator is $\left|\omega t_{i}\right|=\lambda_{i}$, while the numerator is just $n$. Thus $N=n / \lambda_{i}=n_{i}$, showing that for each $t_{i}$ we have

$$
\prod\left(1-\zeta \omega\left(t_{i}\right)\right)=\lambda_{i}^{n^{\prime}},
$$

where the product is taken over those $\zeta \in Z$ such that $\zeta \omega\left(t_{i}\right) \neq 1$.

Then

$$
\begin{aligned}
\prod_{k=1}^{\mu} U_{k}(\omega) & =\prod_{\zeta \in Z} \prod_{i \in \Lambda(\zeta)}\left(1-\zeta \omega\left(t_{i}\right)\right) \\
& =\prod_{i=1}^{n} \prod_{\substack{\zeta \in Z \\
\zeta \omega t_{i} \neq 1}}\left(1-\zeta \omega\left(t_{i}\right)\right)=\prod_{i=1}^{n} \lambda_{i}^{n_{i}} .
\end{aligned}
$$

DEFINITION 4.3. The redundancy $D(\omega)$ of the representation $\omega$ is defined as

$$
D(\omega)=\left(Q(\omega) \cdot U_{2}(\omega) \cdots U_{\mu}(\omega)\right) / F(\omega) .
$$

From Propositions 3.3 and 4.1, it is easy to show Theorem 4.1 and Proposition 4.2.

THEOREM 4.1. $F(\omega)=\left(Q(\omega) U_{1}(\omega) U_{2}(\omega) \cdots U_{\mu}(\omega)\right) / n$, and $D(\omega)=n / U_{1}(\omega)$.

Proposition 4.2. $D(\omega)$ is invariant under r-equivalence, i.e., if $\omega_{1} \sim_{r} \omega_{2}$, then $D\left(\omega_{1}\right)=D\left(\omega_{2}\right)$.

For actual computation of $D(\omega)$, the following proposition is useful.

Proposition 4.3. For $i=1,2, \ldots, \mu$, we denote by $G_{i}$ the subgroup

$$
\left\langle\omega\left(t_{1}\right), \ldots, \omega\left(t_{i-1}\right), \omega\left(t_{i+1}\right), \ldots, \omega\left(t_{\mu}\right)\right\rangle .
$$

Then

$$
U_{1}(\omega)=n^{\mu} /\left(\left|G_{1}\right|\left|G_{2}\right| \cdots\left|G_{\mu}\right|\right)
$$

and hence

$$
D(\omega)=\left(\left|G_{1}\right|\left|G_{2}\right| \cdots\left|G_{\mu}\right|\right) / n^{\mu-1} .
$$

Proof. Let $\Gamma_{1}\left(t_{i}\right)=\left\{\zeta_{j} \in \Gamma_{1}(\omega) \mid \zeta_{j} \omega\left(t_{i}\right) \neq 1\right\}$, with $\Gamma_{1}(\omega)$ as in Definition 4.1. If $\zeta_{j} \in \Gamma_{1}\left(t_{i}\right)$, then $\zeta_{j} \mid G_{i}$ is trivial and hence $\zeta_{j}$ induces an irreducible representation of $\omega\left(A_{\mu}\right) / G_{i}$. Therefore

$$
\left|\Gamma_{1}\left(t_{i}\right)\right|=\left|\frac{\omega\left(A_{\mu}\right)}{G_{i}}\right|=\frac{n}{\left|G_{i}\right|} \text { and } \prod_{\zeta, \in \Gamma_{1}\left(t_{i}\right)}\left(1-\zeta_{j} \omega\left(t_{i}\right)\right)=\frac{n}{\left|G_{i}\right|},
$$

which proves (4.3); (4.4) follows from Definition 4.3.

THEOREM 4.2. $D(\omega)$ is a positive integer and a divisor of $n$. 
Proof. Recalling $m_{i}=\omega t_{i}$, we set $H_{i}=\left\langle m_{i}\right\rangle$ and $H_{i}^{+}=\prod_{j=i+1}^{\mu} H_{j}$, with $H_{\mu}^{+}=1$. By induction on $\mu$, we see that

$$
n=\left|\omega\left(A_{\mu}\right)\right|=\prod_{i=1}^{\mu} \frac{\left|H_{i}\right|}{\left|H_{i} \cap H_{i}^{+}\right|} .
$$

Note also that

$$
\frac{n}{\left|G_{i}\right|}=\frac{\left|H_{i} G_{i}\right|}{\left|G_{i}\right|}=\frac{\left|H_{i}\right|}{\left|H_{i} \cap G_{i}\right|}
$$

By (4.4) we see that

$$
D(\omega)=n \prod_{i=1}^{\mu}\left(\frac{\left|G_{i}\right|}{n}\right)=\prod_{i=1}^{\mu}\left(\frac{\left|H_{i}\right|}{\left|H_{i} \cap H_{i}^{+}\right|}\right) \cdot \prod_{i=1}^{\mu}\left(\frac{\left|H_{i} \cap G_{i}\right|}{\left|H_{i}\right|}\right),
$$

whence

$$
D(\omega)=\prod_{i=1}^{\mu}\left(\frac{\left|H_{i} \cap G_{i}\right|}{\left|H_{i} \cap H_{i}^{+}\right|}\right)
$$

Now since $H_{i} \cap H_{i}^{+}$is a subgroup of $H_{i} \cap G_{i}$ for $i=1,2, \ldots, \mu$, each factor of the last product is an integer, and thus $D(\omega)$ is an integer. Further, since $U_{1}(\omega)=$ $\prod_{i=1}^{\mu}\left|\omega\left(A_{\mu}\right) / G_{i}\right|$ is an integer, $D(\omega)$ must be a divisor of $n$.

Corollary 4.2.1. $D(\omega)=1$ iff $\omega\left(A_{\mu}\right)$ is the direct product $H_{1} \times H_{2} \times \cdots \times H_{\mu}$.

Proof. Sufficiency follows from (4.5) and the fact that $H_{i} \cap G_{i}=1$.

To prove necessity: From (4.5) we see that if $D(\omega)=1$ then $\left|H_{\mu} \cap G_{\mu}\right|=1$. Since the same argument would hold for any index $i$ as well as for index $\mu,\left|G_{i} \cap H_{i}\right|=1$ for all $i$. Therefore $\omega A_{\mu}$ is the direct product $H_{1} \times H_{2} \times \cdots \times H_{\mu}$.

THEOREM 4.3. If $\mu=1$, then $D(\omega)=1$.

Proof. Immediate from (4.5) since $G_{1}=H_{1}^{+}=1$.

THEOREM 4.4. If $\mu=2, D(\omega)=\lambda_{1} \cdot \lambda_{2} / n$.

Proof. Immediate from (4.4) since $\left|G_{1}\right|=\left|H_{2}\right|=\lambda_{2}$ and $\left|G_{2}\right|=\left|H_{1}\right|=\lambda_{1}$.

THEOREM 4.5. If $\omega A_{\mu}$ is a cyclic group of order $n$, let $\lambda_{i}=\left|\omega\left(t_{i}\right)\right|$ and $n_{i}=n / \lambda_{i}$. Define $g_{i}=$ g.c.d. $\left(n_{1}, n_{2}, \ldots, \hat{n}_{i}, \ldots, n_{\mu}\right)$. Then $D(\omega)=n / g_{1} g_{2} \cdots g_{\mu}$.

Proof. Because $n /\left|G_{i}\right|=g_{i}$.

Corollary 4.5.1. If $\omega: A_{\mu} \rightarrow \mathcal{S}\left(J_{n}\right)$ is a cyclic representation such that at least two of the $m_{i}$ are of order $n$, then $D(\omega)=n$.

COROLlARY 4.5.2. If $\mu \geqslant 2$ and $m_{1}^{\varepsilon_{1}}=m_{2}^{\varepsilon_{2}}=\cdots=m_{\mu}^{\varepsilon_{\mu}}$, with each $\varepsilon_{i}= \pm 1$, then $D(\omega)=n$.

Corollary 4.5.3. If $\mu \geqslant 2$ and $m_{1}=m_{2}=\cdots=m_{\mu}$, then $D(\omega)=n$. (This very special case of Corollary 4.5.1 is exactly what is needed to prove the algebraic part of the theorem of Hosokawa and Kinoshita [17]; see §11.) 
We have another formulation of $D(\omega)$.

THEOREM 4.6. For each generator $m_{i}$ of $\mathscr{\Re}$, let $r_{i}$ be the smallest positive power of $m_{i}$ such that $m_{i}^{r_{i}} \in G_{i}$. Define $G^{*}=\left\langle m_{1}^{r_{1}}, \ldots, m_{\mu}^{r_{\mu}}\right\rangle \subset \omega\left(A_{\mu}\right)$. Then $D(\omega)=\left|G^{*}\right|$.

Proof. Since $m_{i}^{r_{i}} \in G_{i},\left|H_{i} \cap G_{i}\right|=\lambda_{i} / r_{i}$, where $\lambda_{i}=\left|m_{i}\right|$. Therefore

$$
G_{i} /\left(H_{i} \cap G_{i}\right) \simeq\left(H_{i} \cdot G_{i}\right) / H_{i}
$$

implies $\left|G_{i}\right| \cdot r_{i}=n$, and hence $D(\omega)=n / r_{1} r_{2} \cdots r_{\mu}$, which is obviously $\left|G^{*}\right|$.

COROllary 4.6.1. $D(\omega)=n$ iff all $r_{i}=1$.

$D(\omega)=1$ iff $\prod_{i=1}^{\mu} r_{i}=n$.

$D(\omega)<n$ iff some $r_{i}>1$.

REMARK. The elements of $G^{*}$ are precisely the elements of $\Re$ for which no single generator $m_{i}$ is indispensable; this is the reason for the choice of the name "redundancy" for the function $D(\omega)$.

5. Product formulae for the redundancy $D(\omega)$. Given two representations $\omega_{1}$ : $A_{\mu} \rightarrow \delta\left(J_{n_{1}}\right)$ and $\omega_{2}: A_{\mu} \rightarrow \delta\left(J_{n_{2}}\right)$, we define the product representation $\omega_{1} \times \omega_{2}$ : $A_{\mu} \rightarrow \varsigma\left(J_{n_{1}} \times J_{n_{2}}\right)$ by

$$
\left(\omega_{1} \times \omega_{2}\right)(g)(i, j)=\left(\omega_{1}(g) i, \omega_{2}(g) j\right) \quad \text { for } i \in J_{n_{1}}, j \in J_{n_{2}} .
$$

Although the transitivity of $\omega_{1}$ and $\omega_{2}$ does not in general imply that of $\omega_{1} \times \omega_{2}$, in most cases discussed in this section the transitivity of $\omega_{1} \times \omega_{2}$ will follow from those of $\omega_{1}$ and $\omega_{2}$.

We would like to find simple relationships between $D\left(\omega_{1}\right), D\left(\omega_{2}\right)$, and $D\left(\omega_{0}\right)$, where $\omega_{0}$ denotes $\omega_{1} \times \omega_{2}$. The following example shows that $D\left(\omega_{0}\right)$ need not equal $D\left(\omega_{1}\right) \cdot D\left(\omega_{2}\right)$ unless additional conditions are imposed on $\omega_{1}$ and $\omega_{2}$.

Example. Let $\omega_{1}: A_{1} \rightarrow \delta\left(J_{2}\right)$ and $\omega_{2}: A_{2} \rightarrow \delta\left(J_{4}\right)$ be defined by

$$
\omega_{1}:\left\{\begin{array}{c}
t_{1} \rightarrow(12) \\
t_{2} \rightarrow(1)(2)
\end{array}\right\} \quad \text { and } \quad \omega_{2}:\left\{\begin{array}{c}
t_{1} \rightarrow(13)(24) \\
t_{2} \rightarrow(1234)
\end{array}\right\} \text {. }
$$

Then by Theorem 4.4, $D\left(\omega_{1}\right)=1$ and $D\left(\omega_{2}\right)=2$, while $D\left(\omega_{1} \times \omega_{2}\right)=1$.

There are two special cases in which the simple product formula can be proven; first, when $\omega_{1} \times \omega_{2}$ is a direct product (defined below); and second, when g.c.d. $\left(n_{1}, n_{2}\right)=1$. We introduce some additional notation to facilitate the proofs.

Let $G_{i j}(i=0,1,2 ; j=1,2, \ldots, \mu)$ denote the subgroup of $\omega_{i}\left(A_{\mu}\right)$ that is generated by the $\mu-1$ elements $\omega_{i}\left(t_{1}\right), \ldots, \widehat{\omega_{i}\left(t_{j}\right)}, \ldots, \omega_{i}\left(t_{\mu}\right)$.

Then Proposition 4.3 gives

$$
D\left(\omega_{i}\right)=\left(\prod_{j=1}^{\mu}\left|G_{i j}\right|\right) / n_{i}^{\mu-1},
$$

and of course $n_{0}=n_{1} n_{2}$. Thus

$$
\frac{D\left(\omega_{1}\right) \cdot D\left(\omega_{2}\right)}{D\left(\omega_{0}\right)}=\prod_{j=1}^{\mu} \frac{\left|G_{1 j}\right|\left|G_{2 j}\right|}{\left|G_{0 j}\right|} .
$$


Definition 5.1. Consider transitive representations $\omega_{1}, \omega_{2}$, and $\omega_{0}=\omega_{1} \times \omega_{2}$, as above. Then $\omega_{0}$ is called the direct product of $\omega_{1}$ and $\omega_{2}$ if each $t_{k}$ is trivialized by at least one of $\omega_{1}, \omega_{2}$. Then the proof of the following proposition is straightforward.

Proposition 5.1. The representation $\omega: A_{\mu} \rightarrow \delta\left(J_{n}\right)$ is the direct product of two representations $\omega_{1}: A_{\mu} \rightarrow \delta\left(J_{n_{1}}\right)$ and $\omega_{2}: A_{\mu} \rightarrow \delta\left(J_{n_{2}}\right)$ if and only if the group $\omega\left(A_{\mu}\right)$ is a direct product of the groups $\omega\left(A_{S_{1}}\right)$ and $\omega\left(A_{S_{2}}\right)$, with $S_{1} \cup S_{2}$ a partition of $J_{\mu}$, where $A_{S_{j}}$ is the subgroup generated by $\left\{t_{k} \mid k \in S_{j}\right\}$.

Theorem 5.1 (The Direct Product Formula). If $\omega_{0}$ is a direct product $\omega_{1} \times \omega_{2}$, then $D\left(\omega_{0}\right)=D\left(\omega_{1}\right) \cdot D\left(\omega_{2}\right)$.

Proof. For $j \in S_{1}$ we have $\left|G_{1 j}\right|=\left|G_{0 j}\right|$ and $\left|G_{2 j}\right|=1$; for $j \in S_{2}$ we have $\left|G_{1 j}\right|=1$ and $\left|G_{2 j}\right|=\left|G_{0 j}\right|$. In either case, $\left|G_{0 j}\right|=\left|G_{1 j}\right| \cdot\left|G_{2 j}\right|$, and the theorem follows from (5.1) above.

Corollary 5.1.1. $D(\omega)=1$ if and only if $\omega$ is a direct product of $\omega_{1}, \omega_{2}, \ldots, \omega_{\mu}$, where $\omega_{i}$ is a representation $A_{\mu} \rightarrow \delta\left(J_{n_{i}}\right)$ such that $\omega_{i}\left(t_{j}\right)=1$ for $i \neq j$.

Proof. This result follows from Proposition 5.1 and Corollary 4.2.1, or from Theorem 4.6.

Theorem 5.2 (The Second Product Formula). If the transitive representation $\omega_{0}$ is the product (not necessarily the direct product) $\omega_{1} \times \omega_{2}$, where $\omega_{i}: A_{\mu} \rightarrow \delta\left(J_{n_{i}}\right)$ are representations with g.c.d. $\left(n_{1}, n_{2}\right)=1$, then $D\left(\omega_{0}\right)=D\left(\omega_{1}\right) \cdot D\left(\omega_{2}\right)$.

Proof. With notation as above, $\left|G_{1 j}\right|$ divides $n_{1},\left|G_{2 j}\right|$ divides $n_{2}$, so g.c.d. $\left(\left|G_{1 j}\right|,\left|G_{2 j}\right|\right)=1$. Consequently $\left|G_{0 j}\right|=\left|G_{1 j}\right| \cdot\left|G_{2 j}\right|$ for all $j=1,2, \ldots, \mu$, and again the theorem follows from (5.1).

With the aid of the Second Product Formula, the calculation of $D(\omega)$ can be accomplished by calculating the redundancy of each restriction of $\omega$ to a Sylow subgroup of $\mathfrak{T}$.

In particular, if $\omega\left(A_{\mu}\right)$ is an elementary abelian $p$-group, $D(\omega)$ is easily calculated as follows.

Proposition 5.2. Let $\omega: A_{\mu} \rightarrow \delta\left(J_{n}\right)$ be a representation such that $\omega\left(A_{\mu}\right)$ is an elementary abelian p-group of rank $m$ generated by $u_{1}, u_{2}, \ldots, u_{m}$.

Let $\omega\left(t_{i}\right)=u_{1}^{\alpha_{i 1}} u_{2}^{\alpha_{i 2}} \cdots u_{m}^{\alpha_{i m}}, i=1,2, \ldots, \mu, 0 \leqslant \alpha_{i j}<p$.

Let $M=\left\|\alpha_{i j}\right\|_{1 \leqslant i \leqslant \mu, 1 \leqslant j \leqslant m}$ and $\tilde{M}_{i}$ the matrix obtained from $M$ by deleting the ith row. Consider $M$ and $\tilde{M}_{i}$ as matrices over the field $\mathbf{Z} / p \mathbf{Z}$.

Let $\nu_{i}$ be the rank of $\tilde{M}_{i}$ over $\mathbf{Z} / p \mathrm{Z}$ :

Then

$$
D(\omega)=p^{\nu_{1}+\nu_{2}+\cdots+\nu_{\mu}-(\mu-1) m} .
$$

In particular, if $m=\mu$, then $D(\omega)=1$. 


\section{ChAPTER II}

6. The modified over presentation of the group of a link. We introduce some notation which will be used in the remainder of this paper.

$L=K_{1} \cup K_{2} \cup \cdots \cup K_{\mu}$ denotes a link of $\mu$ components in $S^{3}$, and $G(L)$ is the link group of $L$, i.e., the fundamental group of $S^{3}-L$.

$P(G)=\left\langle x_{k l}: r_{i j}\right\rangle_{\phi}$ is an over presentation of $G(L)$, as described in [13], where $i, k=1,2, \ldots, \mu ; l=1,2, \ldots, \nu_{k} ; j=1,2, \ldots, \nu_{i} ; x_{k, l}$ represents a meridian of $K_{k}$; and $r_{i j}$ is of the form

$$
r_{i j}=\rho_{i j} u_{i j}^{-1} x_{i j} u_{i j} x_{i, j+1}^{-1} \rho_{i j}^{-1},
$$

where $\rho_{i j}$ and $u_{i j}$ are words in the symbols $x_{k l}$.l.

Denote by $\psi$ the abelianization of $G(L)$; that is, $\psi$ is the natural homomorphism from $G(L)$ onto $G(L) / G(L)^{\prime} \simeq A_{\mu}$, with $x_{k l}^{\psi \phi}=t_{k} \cdot \psi$ can be extended to a ring homomorphism from $\mathbf{Z} G(L) \rightarrow \mathbf{Z} A_{\mu}$. For simplicity, we use the same symbol $\psi$ for this ring homomorphism.

If $\pi: G(L) \rightarrow S\left(J_{n}\right)$ is a representation of $G(L)$, with $\pi G(L)$ abelian and transitive, then we can find a unique map $\omega: A_{\mu} \rightarrow \mathcal{S}\left(J_{n}\right)$ such that $\omega \psi=\pi$. We use the notation $m_{i}=\omega t_{i}$ for $i=1,2, \ldots, \mu$, and $\mathscr{N}=\omega A_{\mu}$ as before.

$U_{\pi}(L)$ and $M_{\pi}(L)$ are, respectively, the unbranched covering space of $S^{3}-L$ and the branched covering space of $S^{3}$ along $L$ determined by $\pi$, or equivalently by $\omega$, or by the sequence of elements $m_{1}, m_{2}, \ldots, m_{\mu} \subset S\left(J_{n}\right)$. श is the monodromy-group of those coverings. As before, $\tau: \varsigma\left(J_{n}\right) \rightarrow \operatorname{GL}(n, \mathbf{Z})$ is a regular matrix representation, and $Z_{\pi}$ (or simply $Z$ ) will denote the set $\zeta_{1}, \zeta_{2}, \ldots, \zeta_{n}$ of irreducible representations of $\mathscr{N}$ into $\varrho$, with $\zeta_{1}$ the trivial representation.

The first lemma we prove in this section is

LEMMA 6.1. $\left(\sum_{i, j}\left(\partial r_{i, j} / \partial x_{k, l}\right)\right)^{\psi \phi}=0$ for each $k, l$.

Proof. Let $\hat{P}(G)=\left\langle y_{i, j}: q_{k, l}\right\rangle$ be the dual presentation to $P(G)$ (see [13]). Then by the fundamental formula of the free differential calculus, we have

$$
\sum_{i, j}\left(\frac{\partial q_{k l}}{\partial y_{i j}}\right)^{\psi \phi}\left(y_{i j}^{\psi \phi}-1\right)=0 \text {. }
$$

However, since $\hat{P}(G)$ is dual to $P(G)$,

$$
{\overline{\left(\frac{\partial q_{k l}}{\partial y_{i j}}\right)^{\psi \phi}} \overline{\left(y_{i j}-1\right)}}^{\psi \phi}=\left(\frac{\partial r_{i j}}{\partial x_{k, l}}\right)^{\psi \phi}\left(x_{k, l}-1\right)^{\psi \phi},
$$

where the bar denotes the isomorphism of $\mathbf{Z} A_{\mu}$ induced by the isomorphism $g \rightarrow g^{-1}$ of $A_{\mu}$. Therefore

$$
\sum_{i, j}\left(\frac{\partial r_{i j}}{\partial x_{k l}}\right)^{\psi \phi}\left(x_{k, l}-1\right)^{\psi \phi}=0 .
$$

Since $A_{\mu}$ is free abelian, $\mathbf{Z} A_{\mu}$ has no divisors of zero, and $t_{k} \neq 1$, so that

$$
\sum_{i, j}\left(\frac{\partial r_{i j}}{\partial x_{k l}}\right)^{\psi \phi}=0
$$


Using $r_{i, j}$ we introduce new relators $R_{i}$ and $R_{i, j}$.

(6.2) $R_{i}=r_{i, 1} r_{i, 2} \cdots r_{i, \nu_{i}}, i=1,2, \ldots, \mu$.

(6.3) For $j=1,2, \ldots, \nu_{i}-1$, let $w_{i, j}=u_{i, j} u_{i, j+1} \cdots u_{i, \nu_{i}-1}$ and define

$$
R_{i j}=x_{i j} w_{i j} x_{i, \nu_{i}}^{-1} w_{i j}^{-1}, \quad i=1,2, \ldots, \mu .
$$

Let $F$ be the free group generated by $x_{k, l}$. Then obviously $\left\langle R_{i, j}, R_{i}\right\rangle^{F}=\left\langle r_{i, j}\right\rangle^{F}$, and hence $\left\{R_{i, j}, R_{i}\right\}$ forms a complete set of relators for $G(L)$.

Next, replace the old generators $x_{k, l}$ by new generators $\xi_{k}, a_{k, l}$, with $\xi_{k}=x_{k, v_{k}}$ and $a_{k, l}=x_{k, l} \xi_{k}^{-1}$, and rewrite $R_{i}, w_{i, j}$ and $R_{i, j}$ in terms of these generators; thus

$$
R_{i, j}=a_{i j} \xi_{i} w_{i j}(\xi, a) \xi_{i}^{-1} w_{i j}(\xi, a)^{-1} .
$$

Thus, we have a new presentation $P^{\prime}(G(L))$ for $G(L)$ :

$$
P^{\prime}(G(L))=\left\langle\xi_{i}, a_{k, l}: R_{i}, R_{i, j}\right\rangle .
$$

We need a further modification for $P(G(L))$.

Given an element $w=\sum_{i=1}^{m} a_{i} h_{i}$ in $\mathbf{Z} F$, where $a_{i} \in \mathbf{Z}$, and $h_{i} \in F$, we write

$$
g^{w}=\prod_{i=1}^{m} h_{i} g^{a_{i}} h_{i}^{-1} .
$$

(Although $g^{w}$ is not determined uniquely by $w$, the ambiguity does not invalidate the following argument.)

Denote $\left(\partial R_{i} / \partial a_{k l}\right) \in \mathbf{Z} F$ by $v(i, k, l)$.

Define

$$
S_{i}=R_{i} \prod_{j=1}^{\mu}\left(R_{j, 1}^{-v(i, j, 1)} \cdots R_{\left.j, \nu_{,}-1, j, \nu_{i}-1\right)}^{-v(i,}\right.
$$

Since $\left\langle R_{i, j}, S_{i}\right\rangle^{F}=\left\langle R_{i, j}, R_{i}\right\rangle^{F}$, where $F=\left\langle\xi_{i}, a_{i, j}\right\rangle$ we obtain the following proposition.

PROPOSITION 6.1. $G(L)$ has a presentation

$$
P^{*}(G)=\left\langle\xi_{1}, \ldots, \xi_{\mu}, a_{k, l} \mid S_{1}, \ldots, S_{\mu}, R_{i, j}\right\rangle,
$$

where $1 \leqslant k, i \leqslant \mu ; l=1,2, \ldots, \nu_{k}-1$; and $j=1,2, \ldots, \nu_{i}-1$.

$P^{*}(G)$ will be called a modified over presentation of $G(L)$.

The Jacobian matrix $J(P)$ of a presentation $P=\langle x: r\rangle_{\phi}$ is the matrix of free derivatives mapped into the group presented by $P$. Thus

$$
J(P)=\left(\partial r_{j} / \partial x_{k}\right)^{\phi} .
$$

The Alexander matrix $A(P)$ of the same presentation $P$ is the abelianization of $J(P)$; thus $A(P)=J(P)^{\psi}$.

It is known (see for example [6]) that Alexander matrices of isotopic links are equivalent, so that the equivalence-class of $A\left(P^{*}\right)$ is an invariant of the link $L$.

Let $\nu$ denote $\nu_{1}+\nu_{2}+\cdots+\nu_{\mu}$. 
Proposition 6.2. The Alexander matrix $A\left(P^{*}\right)$ of the modified over presentation $P^{*}$ is a $\nu \times \nu$ matrix over $\mathbf{Z} A_{\mu}$ which satisfies the following.

(1) $\xi_{k}^{\psi \phi}=t_{k}$ and $\xi_{k}$ represents a meridian of $K_{k}$.

(2) $a_{k, l}^{\phi} \in G^{\prime}(L)$ (i.e. $\left.a_{k l}^{\psi \phi}=1\right)$.

(3) $\left(\partial R_{i, j} / \partial a_{k, l}\right)^{\psi \phi} \equiv \delta_{i k} \cdot \delta_{j l} \bmod \left(1-t_{i}\right)$.

(4) $\left(\partial S_{i} / \partial a_{k, l}\right)^{\psi \phi} \equiv 0 \bmod \left(1-t_{i}\right)$.

(5) $\left(\partial S_{i} / \partial \xi_{k}\right)^{\psi \phi} \equiv 0 \bmod \left(1-t_{i}\right)$ for $i \neq k$.

(6) $\sum_{i=1}^{\mu}\left(\partial S_{i} / \partial \xi_{k}\right)^{\psi \phi}=0$.

(7) $\sum_{k=1}^{\mu}\left(\partial R_{i j} / \partial \xi_{k}\right)^{\psi \phi}\left(1-t_{k}\right)=0$.

(8) $\sum_{i=1}^{\mu}\left(\partial S_{i} / \partial a_{k l}\right)^{\psi \phi}=0$.

Proof. (1) and (2) are obvious. Direct calculations prove (3)-(6). (7) follows from the fundamental formula of the free differential calculus. Finally, (8) is a consequence of Lemma 6.1.

7. Relation matrices for covering spaces. Let $P_{0}(G)=\left\langle x_{k}: r_{j}\right\rangle_{\phi}$ be any presentation of $G(L)$. Let $\pi: G(L) \rightarrow \varsigma\left(J_{n}\right)$ be a (transitive) permutation representation of $G(L)$ and $\tau: S\left(J_{n}\right) \rightarrow \operatorname{GL}(n, \mathbf{Z})$ be a regular matrix representation of $\delta\left(J_{n}\right)$. Then $\tau$ can be extended to a ring homomorphism, also denoted by $\tau$, from $\mathbf{Z S}\left(J_{n}\right) \rightarrow \operatorname{GL}(n, \mathbf{Z})$.

Let $J\left(P_{0}\right)$ denote the Jacobian matrix $\left(\partial r_{j} / \partial x_{k}\right)^{\phi}$ of $P_{0}(G)$. Let $\nu$ be the number of relators $r_{j}$. Then the following is proved in [7].

Proposition 7.1. Let $M^{\prime}$ and $M$ be, respectively, relation matrices of $H_{1}\left(U_{\pi}(L)\right)$ and $H_{1}\left(M_{\pi}(L)\right)$. Then $J\left(P_{0}\right)^{\tau \pi}$ is equivalent to $\left[M^{\prime} O_{*, n-1}\right]$ and

$$
\left[\begin{array}{c}
J\left(P_{0}\right)^{\tau \pi} \\
R_{1}
\end{array}\right]
$$

is equivalent to [M $\left.O_{*, n-1}\right]$, where $R_{1}$ is a so-called branch relation matrix (see [7]).

Now we assume

(7.1) the first $\mu$ generators $x_{1}, \ldots, x_{\mu}$ of $P_{0}(G)$ represent meridians of the knots $K_{1}, \ldots, K_{\mu}$, respectively.

Let $B_{i}$ be the standard representation matrix of Definition 3.1. Let $n_{i}$ be the number of rows of $B_{i}$ and let $n_{\pi}=\sum_{i=1}^{\mu} n_{i}$. Then it is known that the branch relation matrix $R_{1}$ in Proposition 7.1 is given by $\left[B_{1} \oplus \cdots \oplus B_{\mu} O_{n_{\pi}, n \nu-n \mu}\right.$ ].

Let $D\left(P_{0}\right)=\operatorname{diag}\left\{1-x_{1}^{\phi}, \quad 1-x_{2}^{\phi}, \ldots, 1-x_{\mu}^{\phi}\right\} \oplus I_{\nu-\mu}$. Define $\tilde{J}\left(P_{0}\right)=$ $J\left(P_{0}\right) D\left(P_{0}\right)$ to be the reduced Jacobian matrix of the presentation $P_{0}$.

(7.2) For $i=1,2, \ldots, \mu$, the $i$ th column of $J\left(P_{0}\right)$ is multiplied by $\left(1-x_{i}^{\phi}\right)$ to get the $i$ th column of $\tilde{J}\left(P_{0}\right)$; other columns are unchanged.

Then we have the following important theorem.

Theorem 7.1 (The Implicit Branch Relation Theorem). $\tilde{J}\left(P_{0}\right)^{\tau \pi}$ is equivalent to $\left[M O_{*, n-1+n_{\pi}}\right]$, with $M$ as above.

Proof. Write $J\left(P_{0}\right)^{\tau \pi}=N$. Then by Proposition 7.1,

$$
\left(\begin{array}{c}
N \\
R_{1}
\end{array}\right) \simeq\left[\begin{array}{ll}
M O_{*, n-1}
\end{array}\right] .
$$


Let $P_{i}$ denote the $n \times n$ permutation matrix $\tau \pi \phi\left(x_{i}\right)$; then $\left(1-x_{i}\right)^{\pi \pi \phi}=I-P_{i}$. We see that $B_{i}\left(I-P_{i}\right)=0$ and $\left(I-P_{i}\right) \cdot B_{i}^{t}=0$. Now $I-P_{i}$ has nullity $n_{i}$ and there exists a nonsingular integer matrix $T_{i}$ such that the $n \times n$ matrix $\left(I-P_{i}\right) T_{i}=U_{i}$ contains exactly $n_{i}$ zero columns; let those be the columns numbered $j_{1}, j_{2}, \ldots, j_{n_{i}}$.

Now we create a new matrix $\tilde{U}_{i}$ from $U_{i}$; for each $k=1,2, \ldots, n_{i}$, we replace the zero vector in column $j_{k}$ by a unit vector with 1 in the $j_{k}$ row and 0 elsewhere. Consider the matrix $\tilde{D}=\tilde{U}_{1} \oplus \cdots \oplus \tilde{U}_{\mu} \oplus I_{n \nu-n \mu}$. Obviously, det $\tilde{D}=1$, and thus $N \simeq N \tilde{D}$ and

$$
\left[\begin{array}{l}
N \\
R_{1}
\end{array}\right] \simeq\left[\begin{array}{c}
N \tilde{D} \\
R_{1} \tilde{D}
\end{array}\right]
$$

It is easy to show that $B_{i} \tilde{U}_{i}$ is an $n_{i} \times n$ matrix of zeros and ones, with a single 1 in each row, and that the columns containing these nonzero entries comprise an identity matrix of order $n_{i}$; it follows that the matrix $N \tilde{D}$, with those $n_{1}+n_{2}$ $+\cdots+n_{\mu}=n_{\pi}$ columns deleted, is equivalent to

$$
\left(\begin{array}{c}
N \tilde{D} \\
R_{1} \tilde{D}
\end{array}\right)
$$

and the theorem follows by noting that

$$
\left[M O_{*, n-1}\right] \simeq\left(\begin{array}{c}
N \\
R_{1}
\end{array}\right) \simeq\left(\begin{array}{c}
N \tilde{D} \\
R_{1} \tilde{D}
\end{array}\right)
$$

and therefore

$$
\left[M O_{*, n-1+n_{\pi}}\right] \simeq N \cdot D\left(P_{0}\right)^{\tau \pi} \simeq \tilde{J}\left(P_{0}\right)^{\tau \pi} .
$$

Now we define the reduced Alexander matrix $\tilde{A}\left(P_{0}\right)$ of any presentation $P_{0}$ of a link group $G$, provided $P_{0}$ satisfies (7.1), as the abelianization of $\tilde{J}\left(P_{0}\right)$; thus

$$
\tilde{A}\left(P_{0}\right)=\tilde{J}\left(P_{0}\right)^{\psi}=J\left(P_{0}\right)^{\psi} \cdot D\left(P_{0}\right)^{\psi}=A\left(P_{0}\right) \cdot D\left(P_{0}\right)^{\psi} .
$$

Applying Theorem 7.1 and the above definitions to the modified over presentation $P^{*}$, we obtain

Corollary 7.1.1. $\tilde{A}\left(P^{*}\right)^{\tau \omega}=A\left(P^{*}\right)^{\tau \omega} \cdot D\left(P^{*}\right)^{\tau \pi}$ is equivalent to $\left[M O_{*, n-1+n_{\pi}}\right]$, and $A\left(P^{*}\right)^{\tau \omega}$ is equivalent to $\left[M O_{*, n-1}\right]$.

We see, by analogy with (7.2) above, that

(7.3) for $i=1,2, \ldots, \mu$, the $i$ th column of $A\left(P_{0}\right)$ is multiplied by $\left(1-x_{i}^{\psi \phi}\right)=$ $\left(1-t_{i}\right)$ to get the $i$ th column of $\tilde{A}\left(P_{0}\right)$; other columns are unchanged.

These theorems have shown that the structure of the first homology group, of an abelian covering of a link, can be computed by an algorithm easily expressible in ordinary algebraic terms (without reference to the topology). "Perform a certain algebraic computation, then delete a certain number of linearly dependent columns." When the branched covering space has a finite homology group (as it often does), we may then compute the order of that group, using theorems of $\$ 3$ which relate elementary divisors of an integer matrix to its eigenvalues. 
8. Properties of the reduced Alexander matrix. Corollary 7.1.1 shows that, when $H_{1}\left(M_{\pi}\right)$ is finite, the order $T\left(M_{\pi}\right)$ can be found, using theorems of Chapter I. However, we must prove some additional properties of the reduced Alexander matrix $\tilde{A}\left(P^{*}\right)$.

Now each row of $A\left(P^{*}\right)$ or $\tilde{A}\left(P^{*}\right)$ corresponds to a relator $S_{i}$ or $R_{i, j}$ and each column to a generator $\xi_{i}$ or $a_{k, l}$. To stress this correspondence, we name the rows after the relators, the columns after the generators, and call $\partial R / \partial y$ the $(R, y)$-entry.

Further, we rearrange rows and columns of $A\left(P^{*}\right)$ so that the first $\mu$ rows are $S_{i}$-rows and the first $\mu$ columns are $\xi_{j}$-columns.

Proposition 8.1. Let $\nu=\sum_{i=1}^{\mu} \nu_{i}$. Then $\tilde{A}\left(P^{*}\right)$ is a $\nu \times \nu$ matrix with the following properties.

(1) The $S_{i}$-row is divisible by $1-t_{i}$;

(2) the $\xi_{k}$-column is divisible by $1-t_{k}$;

(3) the sum of the $S_{i}$-rows is 0 ;

(4) the sum of the $\xi_{k}$-columns is 0.

Proof. (1) follows from Proposition 6.2(4), (5), and (7.2); (2) follows from (7.2); (3) is a consequence of (6) and (8) of Proposition 6.2; and (4) follows from the fundamental theorem of the free differential calculus and (7) of Proposition 6.2.

Let $\pi: G(L) \rightarrow \varsigma\left(J_{n}\right)$ be an abelian representation.

Let

$$
R=\left[\begin{array}{ccc|c}
B_{1} & & 0 & \\
\vdots & \ddots & \vdots & O_{n_{\pi}+n, n \nu-n \mu} \\
0 & & B_{\mu} & \\
I_{n} & \cdots & I_{n} &
\end{array}\right]
$$

Proposition 8.2. $R \tilde{A}\left(P^{*}\right)^{\tau \omega}=0$ and $\tilde{A}\left(P^{*}\right)^{\tau \omega} R^{t}=0$.

Proof. Let $P_{i}=\tau \pi \phi\left(\xi_{i}\right)$. Then $B_{i}\left(I-P_{i}\right)=0$ and $\left(I-P_{i}\right) B_{i}^{t}=0$, and the result follows from (3) and (4) of Proposition 8.1.

Proposition 8.3. Let $\Lambda$ be a subset of $J_{\mu}=\{1,2, \ldots, \mu\}$. Let $A_{\Lambda}$ be the subgroup of $A_{\mu}$ generated by $\left\{t_{j} \mid j \in \Lambda\right\}$. Let $p$ be the homomorphism from $A_{\mu}$ onto $A_{\Lambda}$ defined by

$$
p\left(t_{j}\right)= \begin{cases}t_{j} & \text { if } j \in \Lambda, \\ 1 & \text { if } j \notin \Lambda .\end{cases}
$$

Then

(1) the minor of $\tilde{A}\left(P^{*}\right)^{p}$, obtained by deleting all $S_{j}$-rows and $\xi_{j}$-columns and $R_{j, k}$-rows and $a_{j, k}$-columns for $j \notin \Lambda$, is the modified Alexander matrix $\tilde{A}\left(P_{\Lambda}^{*}\right)$ of the modified presentation $P_{\Lambda}^{*}$ for the sublink $L_{\Lambda}=\cup_{j \in \Lambda} K_{j}$.

(2) Let $\rho$ be any homomorphism of $A_{\Lambda}$ into $\mathcal{C}$. Then the eigenvalues of $\tilde{A}\left(P^{*}\right)^{\rho p}$ consist of the eigenvalues of $\tilde{A}\left(P_{\Lambda}^{*}\right)^{\rho}, \nu-\nu_{\Lambda}-d$ eigenvalues equal to 1 , and $d$ eigenvalues equal to 0 , where $\nu_{\Lambda}=\Sigma_{j \in \Lambda} \nu_{j}$ and $d=\mu-|\Lambda|$. (See $\$ 6$ for definition of $\nu_{j}$.) 
Proof. (1) is obvious from the definitions.

For (2): First, the $S_{j}$-row and $\xi_{j}$-columns are divisible by $\left(1-t_{j}\right)$ - see Proposition 8.1(1), (2). If $j \notin \Lambda$, then $p\left(t_{j}\right)=1$ and thus these rows and columns are 0 . Therefore, $\tilde{A}\left(P^{*}\right)^{\rho p}$ contains $d=\mu-|\Lambda|$ zero rows and columns.

Next, consider the $R_{j, k}$-rows and $a_{j, r}$ columns for $j \notin \Lambda$. The $\left(R_{j, k}, \xi_{r}\right)$-entry of $\tilde{A}\left(P^{*}\right)$ is $\left(\partial R_{j k} / \partial \xi_{r}\right) \cdot\left(1-t_{r}\right)$. Since $\left(\partial R_{j k} / \partial \xi_{r}\right) \equiv 0 \bmod \left(1-t_{j}\right)$ for $j \neq r$, this entry is always divisible by $\left(1-t_{j}\right)$, and thus 0 at $\rho p$. Further, the $\left(R_{j, k}, a_{m, l}\right)$-entry $\equiv \delta_{j m} \delta_{k l} \bmod \left(1-t_{j}\right)$ by Proposition $6.2(3)$, and hence the $\left(R_{j, k}, a_{j, k}\right)$-entry of $\tilde{A}\left(P^{*}\right)^{p}$, which $=1$, is the only nonzero element in the $R_{j k}$-row.

Therefore, $\tilde{A}\left(P^{*}\right)^{p}$ contains an identity matrix of order $\nu-\nu_{\Lambda}-d$, and $d$ zero rows and columns, showing that $\tilde{A}\left(P^{*}\right)^{p}$ is similar to, and also equivalent to, the matrix $\tilde{A}\left(P_{\Lambda}^{*}\right) \oplus I_{\nu-\nu_{\Lambda}-d} \oplus O_{d, d}$. (2) now follows immediately.

9. Abelian representations and Alexander polynomials. Let $\Delta_{L}\left(t_{1}, t_{2}, \ldots, t_{\mu}\right)$ be the Alexander polynomial of $L . \Delta_{L}\left(t_{1}, t_{2}, \ldots, t_{\mu}\right)$ is a "Laurent" polynomial with integer coefficients; i.e., $\Delta_{L}\left(t_{1}, t_{2}, \ldots, t_{\mu}\right)$ is an element of an integral domain $D=$ $\mathbf{Z}\left[t_{1}, t_{1}^{-1}, \ldots, t_{\mu}, t_{\mu}^{-1}\right]$. We write $f_{1} \doteq f_{2}$ if $f_{1}$ and $f_{2}$ in $D$ are associates. Note that the units of $D$ are $\pm t_{1}^{a_{1}} t_{2}^{a_{2}}, \ldots, t_{\mu}^{a_{\mu}}$, with $a_{1}, \ldots, a_{\mu}$, arbitrary integers.

For $\Lambda$ any subset of $\{1,2, \ldots, \mu\}$, we define $L_{\Lambda}=\cup_{j \in \Lambda} K_{j}$.

We define the modified Alexander polynomial of the link $L$, a function $\tilde{\Delta}_{L}\left(v_{1}, v_{2}, \ldots, v_{\mu}\right)$ of $\mu$ complex variables, by setting

$$
\Lambda=\left\{i \mid v_{i} \neq 1\right\}=\left\{j_{\alpha} \text { with } \alpha=1, \ldots, s=|\Lambda|\right\}
$$

and

$$
\tilde{\Delta}_{L}\left(v_{1}, v_{2}, \ldots, v_{\mu}\right)=\Delta_{L_{\Lambda}}\left(v_{j_{1}}, v_{j_{2}}, \ldots, v_{j_{s}}\right) .
$$

The purpose of this section is to describe the product of the eigenvalues of the matrix $\tilde{J}\left(P^{*}\right)^{\tau \pi}=\tilde{A}\left(P^{*}\right)^{\tau \omega}$ in terms of values assumed by the modified Alexander polynomial $\tilde{\Delta}_{L}$, for any abelian representation $\pi: G(L) \rightarrow \delta\left(J_{n}\right)$.

Proposition 9.1. Let $\tilde{M}_{i}$ be the principal minor of $\tilde{A}\left(P^{*}\right)$ obtained by deleting the $S_{i}$-row and the $\xi_{i}$-column. Then

$$
\tilde{M}_{i} \doteq\left(1-t_{1}\right)\left(1-t_{2}\right) \cdots\left(1-t_{\mu}\right) \Delta_{L}\left(t_{1}, t_{2}, \ldots, t_{\mu}\right) \quad \text { if } \mu>1
$$

and

$$
\tilde{M}_{1} \doteq \Delta_{L}\left(t_{1}\right) \quad \text { if } \mu=1 \text {. }
$$

Proof. It is known (see for example [2, Lemma 1.1, p. 164]) that if $\mu>1$, then the principal minor $M_{i}$ of $A\left(P^{*}\right)$, obtained by deleting the $S_{i}$-row and the $\xi_{i}$-column, is an associate of $\left(1-t_{i}\right) \cdot \Delta_{L}\left(t_{1}, t_{2}, \ldots, t_{\mu}\right)$, whence (7.2) shows the result immediately for $\mu>1$.

For $\mu=1$, the result is well known; see [6].

Let $\zeta: \omega A_{\mu} \rightarrow \mathcal{C}$ be an irreducible representation.

Define $\Lambda(\zeta)=\left\{i \mid \zeta\left(m_{i}\right) \neq 1\right\}$, and $s=|\Lambda(\zeta)|$. 
Proposition 9.2. Let $\lambda_{1}, \lambda_{2}, \ldots, \lambda_{\nu}$ be the eigenvalues of $\tilde{A}\left(P^{*}\right)^{\xi \omega}$, where $\left|\lambda_{1}\right| \geqslant$ $\left|\lambda_{2}\right| \geqslant \cdots \geqslant\left|\lambda_{\nu}\right|$. Let $d=\mu-s$.

(1) If $s>1$, then $\lambda_{\nu-d}=\cdots=\lambda_{\nu}=0$ and

$$
\left|\prod_{i=1}^{\nu-d-1} \lambda_{i}\right|=s\left|\prod_{i \in \Lambda(\zeta)}\left(1-\zeta\left(m_{i}\right)\right)\right| \cdot\left|\tilde{\Delta}_{L}\left(\zeta\left(m_{1}\right), \zeta\left(m_{2}\right), \ldots, \zeta\left(m_{\mu}\right)\right)\right| .
$$

(2) If $s=1$, then $\lambda_{\nu-d}=\cdots=\lambda_{\nu}=0$ and

$$
\left|\prod_{i=1}^{\nu-d-1} \lambda_{i}\right|=\left|\tilde{\Delta}_{L}\left(\zeta\left(m_{1}\right), \zeta\left(m_{2}\right), \ldots, \zeta\left(m_{\mu}\right)\right)\right| .
$$

(3) If $s=0$, then $\lambda_{\nu-d+1}=\cdots=\lambda_{\nu}=0$ and

$$
\left|\prod_{i=1}^{\nu-d} \lambda_{i}\right|=\left|\tilde{\Delta}_{L}\left(\zeta\left(m_{1}\right), \zeta\left(m_{2}\right), \ldots, \zeta\left(m_{\mu}\right)\right)\right|=1 .
$$

Proof. From the matrix $\tilde{A}\left(P^{*}\right)$ delete those rows and columns corresponding to those components $K_{i}$ of $L$ with $i \notin \Lambda(\zeta)$; the result is $\tilde{A}\left(P_{\Lambda}^{*}\right)$, the reduced Alexander matrix of the sublink $L_{\Lambda}=\cup_{i \in \Lambda(\zeta)} K_{i}$.

$\tilde{A}\left(P_{\Lambda}^{*}\right)$ is a $\nu_{\Lambda} \times \nu_{\Lambda}$ matrix over $D$, where $\nu_{\Lambda}=\sum_{i \in \Lambda(\zeta)} \nu_{i}$.

Consider $M=\tilde{A}\left(P_{\Lambda}^{*}\right)^{\zeta \omega}$, and the $1 \times \nu_{\Lambda}$ matrix $R=(1 \cdots 10 \cdots 0)$ with ones for the first $s$ entries and zeros elsewhere, where $s=|\Lambda(\zeta)|$. Then Proposition 8.1(3), (4), shows that $R M=0$ and $M R^{t}=0$. We refer to the proof of Theorem 2.1, and note that $F(R)=s$ when $s>0$.

The proof of Proposition 8.3(2) shows that $\tilde{A}\left(P^{*}\right)^{\zeta \omega}$ is similar to, and also equivalent to, $\tilde{A}\left(P_{\Lambda}^{*}\right)^{\zeta \omega} \oplus I_{\nu-\nu_{\Lambda}-d} \oplus O_{d, d}$, so that the eigenvalues of $\tilde{A}\left(P^{*}\right)^{\zeta \omega}$ consist of the eigenvalues of $\tilde{A}\left(P_{\Lambda}^{*}\right)^{\zeta \omega}=M, \nu-\nu_{\Lambda}-d$ ones, and $d$ zeros, where $d=\mu-s$.

Now, by Theorem 2.1(a), $M$ has at least one eigenvalue equal to 0 .

Let $\lambda_{1}, \lambda_{2}, \ldots, \lambda_{\nu_{1}-1}$ be the remaining eigenvalues of $M$. (Note that some $\lambda_{i}$ may be 0 .)

Let $\tilde{M}$ be a principal submatrix of $M$, obtained by deleting one $S_{i}$-row and $\xi_{i}$-column for some $i \in \Lambda(\zeta)$. Then in the proof of Theorem 2.1 it was shown that there exists a $\nu_{\Lambda} \times \nu_{\Lambda}$ integer matrix $X$ such that

$$
X M X^{t}=\left(\begin{array}{cc}
0 & 0 \\
0 & \tilde{M}
\end{array}\right)
$$

and

$$
\prod_{i=1}^{\nu_{A}^{-1}}\left|\lambda_{i}\right|=F(R) \cdot|\operatorname{det} \tilde{M}|=s \cdot|\operatorname{det} \tilde{M}| .
$$

Now if $s>1$, Proposition 9.1 implies

$$
\begin{aligned}
\prod_{i=1}^{\nu_{\Lambda}-1}\left|\lambda_{i}\right| & =s \prod_{k \in \Lambda(\zeta)}\left(1-\zeta\left(m_{k}\right)\right) \cdot \Delta_{L_{\Lambda}}\left(t_{j_{1}}, \ldots, t_{j_{s}}\right)^{\zeta \omega} \\
& =s \prod_{k \in \Lambda(\zeta)}\left|\left(1-\zeta\left(m_{k}\right)\right)\right| \cdot\left|\tilde{\Delta}_{L}\left(\zeta\left(m_{1}\right), \zeta\left(m_{2}\right), \ldots, \zeta\left(m_{\mu}\right)\right)\right| .
\end{aligned}
$$


If $s=1$, then

$$
\prod_{i=1}^{\nu_{\Lambda}-1}\left|\lambda_{\nu}\right|=\Delta_{L_{\Lambda}}\left(t_{j_{1}}\right)^{\zeta \omega}=\left|\tilde{\Delta}_{L}\left(\zeta\left(m_{1}\right), \zeta\left(m_{2}\right), \ldots, \zeta\left(m_{\mu}\right)\right)\right| .
$$

(1) and (2) of Proposition 9.2 are now obtained from the above equations by noting that $\nu-\nu_{\Lambda}-d$ factors, each equal to 1 , constitute both the eigenvalues and the invariant factors of $I_{\nu-\nu_{\Lambda}-d}$.

Finally, if $s=0$, then $M$ is empty and $\left(\tilde{A}\left(P^{*}\right)\right)^{\xi \omega} \simeq O_{\mu, \mu} \oplus I_{\nu-\mu}$. Therefore,

$$
\prod_{i=1}^{\nu-d}\left|\lambda_{i}\right|=1=\left|\tilde{\Delta}_{L}\left(\zeta\left(m_{1}\right), \zeta\left(m_{2}\right), \ldots, \zeta\left(m_{\mu}\right)\right)\right|,
$$

proving result (3).

10. Main theorems. For the abelian representation $\pi: G(L) \rightarrow \delta\left(J_{n}\right)$, denote by $\left|M_{\pi}(L)\right|$ the order of $H_{1}\left(M_{\pi}(L)\right)$. We interpret $\left|M_{\pi}(L)\right|=0$ to mean that $H_{1}\left(M_{\pi}(L)\right)$ is infinite. With $\omega$ and $Z_{\pi}$ as defined in $\S 6$, we have

Lemma 10.1. Let $M=\left(g_{i j}\right)$ be a $\nu \times \nu$ matrix over $\mathbf{Z} A_{\mu}$. Let $\lambda_{1}^{(r)}, \ldots, \lambda_{\nu}^{(r)}$ be the eigenvalues of $M^{\xi, \omega}=\left(\zeta_{r} \omega\left(g_{i j}\right)\right)$. Let $\lambda_{1}, \lambda_{2}, \ldots, \lambda_{n \nu}$ be the eigenvalues of the integer matrix $M^{\tau \omega}=\left(\tau \omega\left(g_{i j}\right)\right)$. Then $\lambda_{1}, \lambda_{2}, \ldots, \lambda_{n v}$ are exactly $\lambda_{1}^{(1)}, \ldots, \lambda_{\nu}^{(1)}$, $\lambda_{1}^{(2)}, \ldots, \lambda_{v}^{(2)}, \ldots, \lambda_{1}^{(n)}, \ldots, \lambda_{v}^{(n)}$ in some order.

Proof. Since all the matrices of the image $\tau$ ฯ commute, there exists a matrix $P$ which simultaneously diagonalizes them all. For that $P$ and for any $g \in A_{\mu}$,

$$
P(\tau \omega(g)) P^{-1}=\operatorname{diag}\left\{\zeta_{1} \omega(g), \ldots, \zeta_{n} \omega(g)\right\} .
$$

Therefore,

$$
\begin{aligned}
(P \oplus \cdots \oplus P) \| & \tau \omega\left(g_{i j}\right) \|(P \oplus \cdots \oplus P)^{-1} \\
= & \left\|\operatorname{diag}\left\{\zeta_{1} \omega\left(g_{i j}\right), \zeta_{2} \omega\left(g_{i j}\right), \ldots, \zeta_{n} \omega\left(g_{i j}\right)\right\}\right\|_{1 \leqslant i, j \leqslant \nu},
\end{aligned}
$$

which by a simultaneous permutation of rows and columns is seen to have the same eigenvalues as $\zeta_{1} \omega(M) \oplus \zeta_{2} \omega(M) \oplus \cdots \oplus \zeta_{n} \omega(M)$; the lemma follows immediately.

Recall that $\Lambda(\zeta)=\left\{j \mid \zeta m_{j} \neq 1\right\}, \Gamma_{k}=\left\{\zeta \in Z_{\pi} \mid \zeta m_{j} \neq 1\right.$ for exactly $k$ distinct values $j\}$,

$$
U_{k}(\omega)=\prod_{\substack{\zeta \in \Gamma_{k} \\ j \in \Lambda(\zeta)}}\left(1-\zeta m_{j}\right), \quad Q(\omega)=\prod_{i=2}^{\mu}\left|\Lambda\left(\zeta_{i}\right)\right|,
$$

and

$$
D(\omega)=\frac{Q(\omega) U_{2}(\omega) \cdots U_{\mu}(\omega)}{n}=\frac{n}{U_{1}(\omega)} .
$$

Then our main theorem is

THEOREM 10.1.

$$
\left|M_{\pi}(L)\right|=D(\omega) \cdot \prod_{\zeta \in Z_{\pi}}\left|\tilde{\Delta}_{L}\left(\zeta m_{1}, \zeta m_{2}, \ldots, \zeta m_{\mu}\right)\right| .
$$


Proof. Define $\lambda_{1}, \lambda_{2}, \ldots, \lambda_{n \nu}$ as the eigenvalues of $M=\left[\tilde{A}\left(P^{*}\right)\right]^{\tau \omega}$, and $e_{1}, e_{2}, \ldots, e_{n \nu}$ as its elementary divisors, with

$$
\left|\lambda_{1}\right| \geqslant\left|\lambda_{2}\right| \geqslant \cdots \geqslant\left|\lambda_{n \nu}\right| \text { and } e_{1}\left|e_{2}, \ldots, e_{n \nu-1}\right| e_{n \nu} .
$$

From Theorem 2.1 and Proposition 8.2, we deduce that

$$
e_{n \nu-\left(n_{\pi}+n-1\right)+1}=\cdots=e_{n \nu}=\lambda_{n \nu-\left(n_{\pi}+n-1\right)+1}=\cdots=\lambda_{n \nu}=0
$$

and

$$
\prod_{i=1}^{n \nu-\left(n_{\pi}+n-1\right)}\left|\lambda_{i}\right|=F(\omega) \prod_{i=1}^{n \nu-\left(n_{\pi}+n-1\right)} e_{i}=F(\omega) \cdot\left|M_{\pi}(L)\right| .
$$

Let $\lambda_{i}^{(r)}$, with $1 \leqslant r \leqslant n$ and $1 \leqslant i \leqslant \nu$, be the eigenvalues defined in Lemma 10.1 with $M=\tilde{A}\left(P^{*}\right)$.

Define

$$
\Omega\left(\zeta_{1}\right)=\prod_{i=1}^{\nu-d_{1}}\left|\lambda_{i}^{(1)}\right|=1 \quad \text { and } \quad \Omega\left(\zeta_{j}\right)=\left|\prod_{i=1}^{\nu-d_{j}-1} \lambda_{i}^{(j)}\right|
$$

for $j=2, \ldots, \mu$, where $d_{j}=\mu-\left|\Lambda\left(\zeta_{j}\right)\right|$.

Then Lemma 10.1 and Proposition 9.2 let us conclude that

$$
\begin{aligned}
\prod_{i=1}^{n \nu-\left(n_{n}+n-1\right)}\left|\lambda_{i}\right| & =\prod_{j=1}^{n} \Omega(\zeta j) \\
& =\prod_{\substack{\zeta \in Z_{\pi} \\
|\Lambda(\zeta)| \geqslant 2}}\left(|\Lambda(\zeta)| \cdot \prod_{i \in \Lambda(\zeta)}\left(1-\zeta m_{i}\right)\right) \cdot \prod_{\zeta \in Z_{\pi}} \tilde{\Delta}_{L}\left(\zeta m_{1}, \zeta m_{2}, \ldots, \zeta m_{\mu}\right) \\
& =Q(\omega) U_{2}(\omega) \cdots U_{\mu}(\omega) \prod_{\zeta \in Z_{\pi}} \tilde{\Delta}_{L}\left(\zeta m_{1}, \zeta m_{2}, \ldots, \zeta m_{\mu}\right),
\end{aligned}
$$

and hence

$$
\begin{aligned}
\left|M_{\pi}(L)\right| & =\frac{Q(\omega) U_{2}(\omega) \cdots U_{\mu}(\omega)}{F(\omega)} \prod_{\zeta \in Z_{\pi}} \tilde{\Delta}_{L}\left(\zeta m_{1}, \zeta m_{2}, \ldots, \zeta m_{\mu}\right) \\
& =D(\omega) \cdot \prod_{\zeta \in Z_{\pi}} \tilde{\Delta}_{L}\left(\zeta \omega t_{1}, \zeta \omega t_{2}, \ldots, \zeta \omega t_{\mu}\right) .
\end{aligned}
$$

Definition 10.1. If $\mu \geqslant 2$, we can define the Hosokawa polynomial $\nabla_{L}(t)$ of the link $L$ as

$$
\nabla_{L}(t)=\Delta_{L}(t, t, \ldots, t) /(1-t)^{\mu-2} .
$$

Then the following corollary seems to be the only special case which has appeared in the literature reflecting a nontrivial $D(\omega)$. (See [17] and $\$ 11$ below.)

Corollary 10.1.1. Let $\pi: G(L) \rightarrow \delta\left(J_{n}\right)$ be a cyclic representation such that $\pi\left(\xi_{i}\right)=(12 \cdots n)$ for all $i$, for a link $L$ with $\mu>1$. Then

$$
\left|M_{\pi}(L)\right|=n^{\mu-1} \prod_{i=1}^{n-1} \nabla_{L}\left(\eta^{i}\right)
$$

where $\eta$ is a primitive nth root of unity. 
Proof. From Theorem 10.1 and Corollary 4.5.3,

$$
\begin{aligned}
\left|M_{\pi}(L)\right| & =n \prod_{\zeta \in Z_{\pi}} \tilde{\Delta}_{L}\left(\zeta \omega t_{1}, \zeta \omega t_{2}, \ldots, \zeta \omega t_{\mu}\right)=n \prod_{i=1}^{n-1} \Delta_{L}\left(\eta^{i}, \ldots, \eta^{i}\right) \\
& =n \prod_{i=1}^{n-1}\left(1-\eta^{i}\right)^{\mu-2} \nabla_{L}\left(\eta^{i}\right)=n^{\mu-1} \prod_{i=1}^{n-1} \nabla_{L}\left(\eta^{i}\right) . \quad \square
\end{aligned}
$$

Finally we consider the special case where $\omega$ is a direct product.

Definition 10.2. We call $\pi: G(L) \rightarrow \delta\left(J_{n}\right)$ the direct product of two representations $\pi_{1}$ and $\pi_{2}$ if the representation $\omega$ induced by $\pi$ is the direct product (Definition 5.1) of representations $\omega_{i}$ induced by $\pi_{i}$ for $i=1,2$.

THEOREM 10.2. If $\pi$ is a direct product of two representations $\pi_{i}: G(L) \rightarrow \delta\left(J_{n_{i}}\right)$, $i=1,2$, and we let $M_{1}$ and $M_{2}$ denote the branched covering spaces of $L$ induced by $\pi_{1}$ and $\pi_{2}$, respectively, then $\left|M_{1}(L)\right| \cdot\left|M_{2}(L)\right|$ divides $\left|M_{\pi}(L)\right|$.

Proof. Since the representation $\omega$ induced by $\pi$ is the direct product of $\omega_{1}, \omega_{2}$, there is a partition $S_{1} \cup S_{2}$ of $J_{\mu}$ such that $\omega_{i}\left(t_{k}\right)=1$ for $t_{k} \notin S_{i}$. We may assume without loss of generality that $S_{1}=\{1,2, \ldots, r\}$ and $S_{2}=\{r+1, \ldots, \mu\}$. Let $L_{i}=$ $\cup_{j \in S_{i}} K_{j}$. Let $Z_{\pi}, Z_{1}, Z_{2}$, respectively, be the sets of irreducible representations of $\omega\left(A_{\mu}\right), \omega_{1}\left(A_{\mu}\right), \omega_{2}\left(A_{\mu}\right)$ into $\mathcal{C}$. Then from the definition of $\tilde{\Delta}_{L}$ it is easy to see that $\prod_{\zeta^{\prime} \in Z_{1}} \tilde{\Delta}_{L_{1}}\left(\zeta^{\prime} \omega_{1}\left(t_{1}\right), \ldots, \zeta^{\prime} \omega_{1}\left(t_{r}\right)\right)$ times $\prod_{\zeta^{\prime \prime} \in Z_{2}} \tilde{\Delta}_{L_{2}}\left(\zeta^{\prime \prime} \omega_{2}\left(t_{r+1}\right), \ldots, \zeta^{\prime \prime} \omega_{2}\left(t_{\mu}\right)\right)$ divides $\prod_{\zeta \in Z_{\pi}} \tilde{\Delta}_{L}\left(\zeta \omega\left(t_{1}\right), \ldots, \zeta \omega\left(t_{\mu}\right)\right)$. Since $\omega$ is a direct product, it follows from Theorem 5.1 that $D(\omega)=D\left(\omega_{1}\right) \cdot D\left(\omega_{2}\right)$ and the result follows.

11. Cyclic coverings of links. This section attempts to clarify the relationships among alternative definitions of " $n$-sheeted cyclic covering of a link $L$ ", each of which generalizes the well-defined notion of "cyclic covering of a knot". The ambiguity has seldom been emphasized, or even described, because each individual paper on coverings of links has usually been concerned with only one definition.

We provide five definitions for cyclic covering of a link, and show that they are all equivalent in case $\mu=1$. Examples show that they are all distinct, even when $\mu=2$. We apply the more specific terms, defined in Definitions 11.1-11.5 below, to either an abelian branched covering $M$ or the associated representation $\omega$.

Hosokawa and Kinoshita proved in [17], by methods much simpler than ours, a result equivalent to our main Theorem 10.1 as specialized to a cyclic covering in the narrowest of these five senses. Their methods and results can in fact be broadened slightly, but our examples show that their methods cannot be extended even as far as the broader definitions of cyclic coverings.

Definition 11.1. $M$ and $\omega$ are strictly-cyclic (or S-cyclic) if every meridian permutes the sheets of $M$ in the same way, i.e., if $m_{1}=m_{2}=\cdots=m_{\mu}$. (This is the definition used in [17].)

DeFINITION 11.2. $M$ and $\omega$ are almost-strictly-cyclic (or AS-cyclic) if $m_{1}^{\varepsilon_{1}}=m_{2}^{\varepsilon_{2}}=$ $\cdots=m_{\mu}^{\varepsilon_{\mu}}$, where every $\varepsilon_{i}= \pm 1$.

DefinItION 11.3. $M$ and $\omega$ are meridian-cyclic (or $\xi$-cyclic) if each meridian of $L$ is covered in $M$ by a single circle, i.e., if $\left\langle m_{1}\right\rangle=\left\langle m_{2}\right\rangle=\cdots=\left\langle m_{\mu}\right\rangle=\mathfrak{N}$. 
Remark. An equivalent definition of $\xi$-cyclic would be $\left|m_{1}\right|=\left|m_{2}\right|=\cdots=$ $\left|m_{\mu}\right|=n$.

Definition 11.4. $M$ and $\omega$ are singly-cyclic if some meridian is covered in $M$ by a single circle, i.e., if some $m_{i}$ has order $n$.

DeFINITION 11.5. $M$ and $\omega$ are monodromy-cyclic (or $\mathfrak{T}$-cyclic) if the monodromygroup $\Re$ is cyclic.

The following propositions are evident from the definitions.

Proposition 11.1. Every S-cyclic covering is AS-cyclic; every AS-cyclic covering is $\xi$-cyclic; every $\xi$-cyclic covering is singly-cyclic and every singly-cyclic covering is NR-cyclic.

Proposition 11.2. Each Th-cyclic covering of a link is $S$-cyclic if either $\mu=1$ (the link is a knot) or $n=2$ and no $m_{i}$ is trivial.

Proposition 11.3. Each T-cyclic covering of a link $L$ is $A S$-cyclic if $n=3$ and no $m_{i}$ is trivial.

Proposition 11.4. If $n$ is square-free, every abelian covering of $n$ sheets is T-cyclic.

Proposition 11.5. If $n$ is a prime power, every N-cyclic covering of $n$ sheets is singly-cyclic.

Proposition 11.6. If $n$ is prime, every abelian covering of $n$ sheets is $\xi$-cyclic if no $m_{i}=1$.

Let us now recall Corollary 10.1.1: If $\mu>1$ and $m_{1}=m_{2}=\cdots=m_{\mu}$, then $\left|M_{\pi}(L)\right|=n^{\mu-1} \Pi_{i=1}^{n-1} \nabla_{L}\left(\eta^{i}\right)$ where $\eta$ is any primitive $n$th root of unity. As mentioned above, that result was proved in [17], and applies only to a strictly-cyclic covering. The methods of [17] could also have been used to give $\left|M_{\pi}(L)\right|$ for an almost-strictly-cyclic covering, without using the heavy machinary of Chapter I of this paper, as shown by

Proposition 11.7. If $\mu>1$, and $\pi$ determines an AS-cyclic covering with $\varepsilon_{i}$ as above, then

$$
\left|M_{\pi}(L)\right|=n^{\mu-1} \cdot \prod_{i=1}^{n-1} \nabla_{L^{\prime}}\left(\eta^{i}\right)
$$

where

$$
\nabla_{L^{\prime}}(t)=\Delta_{L}\left(t^{\varepsilon_{1}}, t^{\varepsilon_{2}}, \ldots, t^{\varepsilon_{\mu}}\right) /(1-t)^{\mu-2}
$$

Proof. Easily verified by applying the result of [17] to the link $L^{\prime}$ constructed from $L$ by reversing the orientation of those $K_{i}$ for which $\varepsilon_{i}=-1$; the AS-cyclic covering of $L$ is then identical to an S-cyclic covering of $L^{\prime}$.

We present as examples four 6-sheeted coverings of the link $L$ shown in Figure 1, and four 9-sheeted coverings of the same link. The examples demonstrate that the five different definitions of "cyclic covering of a link" are indeed all different. 


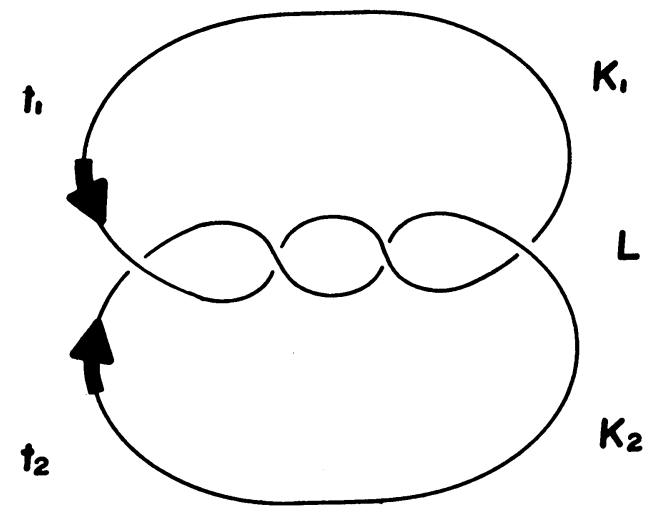

FIGURE 1

The Alexander polynomial of $L$ is $\Delta_{L}\left(t_{1}, t_{2}\right)=1+t_{1} t_{2}$. For simplicity we use $x, y$ for $m_{1}, m_{2}$, respectively. Note also that $\Delta_{K_{1}}\left(t_{1}\right) \equiv 1$, and $\Delta_{K_{2}}\left(t_{2}\right) \equiv 1$; thus $\tilde{\Delta}_{L}\left(t_{1}, t_{2}\right)$ $=1+t_{1} t_{2}$ when $t_{1} \neq 1$ and $t_{2} \neq 1, \tilde{\Delta}_{L}\left(t_{1}, t_{2}\right)=1$. otherwise.

EXAMPLE 1. $\Re=\left\langle x, y \mid x^{2}=y^{3}=1, x y=y x\right\rangle$; with $\eta$ a primitive 6 th root of unity we have

$$
\begin{aligned}
\prod_{\zeta \in Z_{\pi}} \tilde{\Delta}_{L}\left(\zeta m_{1}, \zeta m_{2}\right) & =\prod_{i=0}^{5} \tilde{\Delta}_{L}\left(\eta^{3 i}, \eta^{2 i}\right) \\
& =\prod_{i=1,5} \Delta_{L}\left(\eta^{3 i}, \eta^{2 i}\right)=\left(1-\eta^{2}\right)\left(1-\eta^{4}\right)=3 .
\end{aligned}
$$

From either Theorem 5.1 or Theorem 5.2, we see that $D(\omega)=1$. Thus $\left|M_{\pi}(L)\right|=3$.

EXAMPLE 2. $\Re=\left\langle x, y \mid x=y^{2}, y^{6}=1\right\rangle$; now

$$
\begin{aligned}
\prod_{\zeta \in Z_{\pi}} \tilde{\Delta}_{L}\left(\zeta m_{1}, \zeta m_{2}\right) & =\prod_{i=0}^{5} \tilde{\Delta}_{L}\left(\eta^{2 i}, \eta^{i}\right)=\prod_{i=1,2,4,5} \Delta_{L}\left(\eta^{2 i}, \eta^{i}\right) \\
& =\prod_{i=1,2,4,5}\left(1+\eta^{3 i}\right)=0,
\end{aligned}
$$

while $D(\omega)=3$ by Theorem 4.4; thus $\left|M_{\pi}(L)\right|=0$.

EXAMPLE 3. $\Re=\left\langle x, y \mid x y=1, y^{6}=1\right\rangle$;

$$
\begin{aligned}
\prod_{\zeta \in Z_{\pi}} \tilde{\Delta}_{L}\left(\zeta m_{1}, \zeta m_{2}\right) & =\prod_{i=0}^{5} \tilde{\Delta}_{L}\left(\eta^{-i}, \eta^{i}\right)=\prod_{i=1}^{5} \Delta_{L}\left(\eta^{-i}, \eta^{i}\right) \\
& =\left(1+\eta^{0}\right)^{5}=32 .
\end{aligned}
$$

Here $D(\omega)=6$ by Corollary 4.5.2; thus $\left|M_{\pi}(L)\right|=192$.

EXAMPLE 4. $\Re=\left\langle x, y \mid x=y, y^{6}=1\right\rangle$. Now

$$
\prod_{\zeta \in Z_{\pi}} \tilde{\Delta}\left(\zeta m_{1}, \zeta m_{2}\right)=\prod_{i=0}^{5} \tilde{\Delta}\left(\eta^{i}, \eta^{i}\right)=\prod_{i=1}^{5}\left(1+\eta^{2 i}\right)=2 .
$$

$D(\omega)=6$ by Corollary 4.5.3, so $\left|M_{\pi}(L)\right|=12$. 
Note that all these orders are distinct, so the coverings are surely different 3-manifolds. Also, Example 1 is $\mathfrak{T}$-cyclic but not singly-cyclic, Example 2 is singly-cyclic but not $\xi$-cyclic, Example 3 is AS-cyclic but not S-cyclic, and Example 4 is S-cyclic.

EXAMPLE 5. O $\pi=\left\langle x, y \mid x=y^{3}, y^{9}=1\right\rangle$; with $\eta=\exp (2 i \pi / 9)$,

$$
\begin{aligned}
\prod_{\zeta \in Z_{\pi}} \tilde{\Delta}_{L}\left(\zeta m_{1}, \zeta m_{2}\right) & =\prod_{i=0}^{8} \tilde{\Delta}_{L}\left(\eta^{3 i}, \eta^{i}\right)=\prod^{\prime} \Delta_{L}\left(\eta^{3 i}, \eta^{i}\right) \\
& =\prod^{\prime}\left(1+\eta^{4 i}\right)=1,
\end{aligned}
$$

where the last two products are over the set $i=1,2,4,5,7,8$. Now $D(\omega)=3$ by Theorem 4.4, so $\left|M_{\pi}(L)\right|=3$.

EXAMPLE 6. $\mathfrak{R}=\left\langle x, y \mid x=y^{2}, y^{9}=1\right\rangle$.

$$
\prod_{\zeta \in Z_{\pi}} \tilde{\Delta}_{L}\left(\zeta m_{1}, \zeta m_{2}\right)=\prod_{i=0}^{8} \tilde{\Delta}_{L}\left(\eta^{2 i}, \eta^{i}\right)=\prod_{i=1}^{8}\left(1+\eta^{3 i}\right)=4 .
$$

Now $D(\omega)=9$ by Corollary 4.5.1, so $\left|M_{\pi}(L)\right|=36$.

EXAMPLE 7. $\Re=\left\langle x, y \mid x y=1, y^{9}=1\right\rangle$. Here

$$
\prod_{\zeta \in Z_{\pi}} \tilde{\Delta}_{L}\left(\zeta m_{1}, \zeta m_{2}\right)=\prod_{i=0}^{8} \tilde{\Delta}_{L}\left(\eta^{-i}, \eta^{i}\right)=\prod_{i=1}^{8}\left(1+\eta^{i-i}\right)=256,
$$

while $D(\omega)=9$ by Corollary 4.5.2, so $\left|M_{\pi}(L)\right|=2304$.

EXAMPLE 8. $\Re=\left\langle x, y \mid x=y, y^{9}=1\right\rangle$. In this case

$$
\prod_{\zeta \in Z_{\pi}} \tilde{\Delta}_{L}\left(\zeta m_{1}, \zeta m_{2}\right)=\prod_{i=0}^{8} \tilde{\Delta}_{L}\left(\eta^{i}, \eta^{i}\right)=\prod_{i=1}^{8}\left(1+\eta^{2 i}\right)=1,
$$

while $D(\omega)=9$ by Corollary 4.5.3, so $\left|M_{\pi}(L)\right|=9$.

Note that Example 5 is singly-cyclic but not meridian-cyclic, Example 6 is meridian-cyclic but not almost-strictly-cyclic, Example 7 is almost-strictly-cyclic but not strictly-cyclic, and Example 8 is strictly-cyclic; again, all four coverings are nonhomeomorphic.

These eight examples show that all five definitions of "cyclic covering" are distinct whenever $\mu \geqslant 2$.

12. Z-homology spheres as abelian coverings. As an interesting application of our main theorem, we will consider a $\mathbf{Z}$-homology sphere that is obtained as an abelian covering space of a link. One such example was given by Seifert [21] as follows.

Let $L$ be a torus link of type $(\mu, \mu) . L$ consists of $\mu$ unknotted knots $K_{1}, K_{2}, \ldots, K_{\mu}$. Consider a cyclic representation $\pi_{i}: G\left(K_{i}\right) \rightarrow \mathbf{Z}_{r_{i}} \subset \delta\left(J_{r_{i}}\right)$ for each $i=1,2, \ldots, \mu$. Assume that $r_{1}, r_{2}, \ldots, r_{\mu}$ are pairwise prime. Each $\pi_{i}$ is extended to a representation $\tilde{\pi}_{i}: G(L) \rightarrow \mathbf{Z}_{r_{i}} \subset \delta\left(J_{r_{i}}\right)$ so that the induced representation $\tilde{\omega}_{i}: A_{\mu} \rightarrow \mathbf{Z}_{r_{i}} \subset \delta\left(J_{r_{i}}\right)$ satisfies $\tilde{\omega}_{i}\left(t_{j}\right)=1$ for $j \neq i$. Then the direct product

$$
\tilde{\pi}=\tilde{\pi}_{1} \times \cdots \times \tilde{\pi}_{\mu}: G(L) \rightarrow \mathbf{Z}_{r_{1}} \times \cdots \times \mathbf{Z}_{r_{\mu}} \subset \delta\left(J_{r_{1}} \times \cdots \times J_{r_{\mu}}\right)
$$


is also a cyclic representation. Seifert proved that the branched covering space of $L$ associated with $\tilde{\pi}$ is a $\mathbf{Z}$-homology sphere. (This is also a consequence of Theorem 10.1, since $\Delta_{L}\left(t_{1}, t_{2}, \ldots, t_{\mu}\right)=\left(1-t_{1} \cdot t_{2} \cdots t_{\mu}\right)^{\mu-2}$ and $D(\tilde{\pi})=1$ by Corollary 5.1.1.) A partial converse to this result follows immediately from Corollary 5.1.1. In fact we obtain

THEOREM 12.1. Let $\pi: G(L) \rightarrow \delta\left(J_{n}\right)$ be an abelian representation. If the branched covering space of $L$ associated with $\pi$ is a $\mathbf{Z}$-homology sphere, then the induced abelian representation $\omega: A_{\mu} \rightarrow \delta\left(J_{n}\right)$ must be a direct product of $\mu$ cyclic representations.

Proof. If $M_{\pi}$ is a Z-homology sphere, then $\left|M_{\pi}\right|=1$ and thus $D(\pi)$ must have been equal to 1. By Corollary 5.1.1, $\omega$ must be a direct product.

Theorem 10.2 gives us further restrictions on the covering space, and on the link type $L$. Since $\left|M_{\pi}\right|=1, \pi$ is a direct product $\pi=\pi_{1} \times \pi_{2} \times \cdots \times \pi_{\mu}$. Thus, for any subset $\Lambda=\left\{i_{1}, i_{2}, \ldots, i_{s}\right\}$ of $J_{n}$ with $|\Lambda|=s$, and corresponding sublink $L_{\Lambda}$, we can define a direct product

$$
\pi^{\prime}=\pi_{i_{1}} \times \pi_{i_{2}} \times \cdots \times \pi_{i_{s}}: G\left(L_{\Lambda}\right) \rightarrow \delta\left(J_{r_{i_{1}}} \times J_{r_{i_{2}}} \times \cdots \times J_{r_{i_{s}}}\right) .
$$

Then Theorem 10.2 shows that $\left|M_{\pi^{\prime}}(L)\right|$ divides $\left|M_{\pi}(L)\right|=1$, so $\left|M_{\pi^{\prime}}(L)\right|=1$ also, and the covering space of $L_{\Lambda}$ associated with the restriction $\left.\pi\right|_{G\left(L_{\Lambda}\right)}$ is also a $\mathbf{Z}$-homology sphere. This is a quite severe restriction, and yields still another restriction on the $\pi_{i}$, as given in the following proposition.

Proposition 12.1. Let $\alpha_{i j}$ denote the linking number, $\operatorname{link}\left(K_{i}, K_{j}\right)$, between $K_{i}$ and $K_{j}$. If, for some $i, j$, g.c.d. $\left(r_{i}, \alpha_{i j}\right) \neq 1$, then $M_{\pi}(L)$ cannot be a $\mathbf{Z}$-homology sphere.

Proof. Suppose g.c.d. $\left(r_{i}, \alpha_{i j}\right)=m \neq 1$. Consider $\pi_{0}=\pi_{i} \times \pi_{j}: G\left(K_{i} \cup K_{j}\right) \rightarrow$ $\delta\left(J_{r_{i}} \times J_{r_{j}}\right)$, and the induced $\omega_{0}=\omega_{i} \times \omega_{j}: A_{2} \rightarrow \delta\left(J_{r_{i}} \times J_{r_{j}}\right)$. Denote by $\Delta_{i}(x)$, $\Delta_{j}(x)$ and $\Delta_{0}(x, y)$, respectively, the Alexander polynomials of $K_{i}, K_{j}$ and $K_{i} \cup K_{j}$. Then by Theorem 10.1, we have

$$
\begin{aligned}
\left|M_{\pi_{0}}\right| & =\prod_{\zeta \in Z_{\pi_{0}}} \tilde{\Delta}_{0}\left(\zeta \omega_{0}(x), \zeta \omega_{0}(y)\right) \\
& =\prod_{a=1}^{r_{i}-1} \Delta_{i}\left(\xi^{a}\right) \cdot \prod_{b=1}^{r_{j}-1} \Delta_{j}\left(\eta^{b}\right) \cdot \prod \Delta_{0}\left(\xi^{a}, \eta^{b}\right),
\end{aligned}
$$

where $\xi$ and $\eta$ are, respectively, a primitive $r_{i}$ th and a primitive $r_{j}$ th root of unity, and the last factor is the product over $1 \leqslant a<r_{i}, 1 \leqslant b<r_{j}$.

Let $p$ be a prime divisor of $r_{j}$.

Since $\Delta_{0}(x, 1)=\left(1+x+\cdots+x^{\left|\alpha_{i j}\right|-1}\right) \Delta_{i}(x)($ see [23]), it follows that

$$
\begin{aligned}
\prod_{a, b \neq 0} \Delta_{0}\left(\xi^{a}, \eta^{b}\right) & \equiv \prod_{a=1}^{r_{i}-1} \Delta_{0}\left(\xi^{a}, 1\right) \quad(\bmod p) \\
& =\prod_{a=1}^{r_{i}-1}\left(1+\xi^{a}+\cdots+\xi^{a \cdot\left(\left|\alpha_{i j}\right|-1\right)}\right) \cdot \Delta_{i}\left(\xi^{a}\right) \\
& \equiv 0(\bmod p),
\end{aligned}
$$

and thus $\left|M_{\pi_{0}}\right| \equiv 0(\bmod p)$. 
By combining this with previous results, we obtain

THEOREM 12.2. For $M_{\pi}(L)$ to be a Z-homology sphere, it is necessary that both

(1) $\pi$ is a direct product $\pi=\pi_{1} \times \pi_{2} \times \cdots \times \pi_{\mu}$ of $\mu$ cyclic representations $\pi_{i}$ : $G\left(K_{i}\right) \rightarrow \varsigma\left(J_{r_{i}}\right), 1 \leqslant i \leqslant \mu$, with $r_{i}$ prime to $\operatorname{link}\left(K_{i}, K_{j}\right)$ for any $j(\neq i)$, and

(2) for every subset $\Lambda$ of $J_{n}$, the branched covering space of $L_{\Lambda}$ induced by $\pi$ is a Z-homology sphere.

Consider the torus link $K_{\mu, \mu}$ of type $(\mu, \mu)$. Since each linking number is +1 or -1 , condition (1) of Theorem 12.2 will surely be satisfied by an abelian covering which is a direct product of cyclic coverings of arbitrary orders. Furthermore, every proper sublink of $K_{\mu, \mu}$ is a torus link $K_{\nu, \nu}$ for some $\nu<\mu$, and each component is unknotted, so that condition (2) is also likely to be satisfied. Thus, one might hope to produce a $\mathbf{Z}$-homology sphere by constructing a suitable abelian direct-product covering of $K_{\mu, \mu}$. Indeed, the example given by Seifert in [21] is of this type. However, it is possible to find a link which is not a torus link of type $(\mu, \mu)$ and which nevertheless has a $\mathbf{Z}$-homology sphere as an abelian covering; we provide an example $L$.

Let $K_{0}$ be the figure-eight knot and let $\phi: G\left(K_{0}\right) \rightarrow D_{5} \subset \mathcal{S}\left(J_{10}\right)$ be the maximal (regular) dihedral representation of $G\left(K_{0}\right)$. Such a representation exists. Then the branched covering space $M_{\phi}$ of $K_{0}$ associated with $\phi$ is a 3-sphere [3] and $K_{0}$ is covered by a link $L_{0}$ of 5 components, each of which is unknotted. Any three or fewer components of $L_{0}$ form a torus link of type $(k, k)$ but the 4-component sublinks of $L_{0}$, though all isotopic, are not torus links. We choose for $L$ one of those 4-component sublinks. Since $\Delta_{L}\left(t_{1}, t_{2}, t_{3}, t_{4}\right)=\left(t_{1} t_{2}-t_{3} t_{4}\right)\left(t_{1} t_{3}-t_{2} t_{4}\right)$, it is easy to show that $\left|H_{1}\left(M_{\pi}\right)\right|=1$ for the abelian direct product $\pi=\pi_{1} \times \pi_{2} \times \pi_{3} \times \pi_{4}$ : $\rightarrow \varsigma\left(J_{2} \times J_{3} \times J_{5} \times J_{7}\right)$. (We thank Professor Burde for suggesting this example.)

It seems probable that suitable abelian coverings of $L_{0}$ may also produce $\mathbf{Z}$ homology spheres. Indeed, we conjecture that $\mathbf{Z}$-homology spheres may occur as abelian coverings of the link $L$ produced by the above construction, even using an arbitrary two-bridged knot $K$ in place of the figure-eight knot.

Since any two components of such an $L$ have linking number +1 or -1 [4], condition (1) in Theorem 12.2 is surely satisfied. We also note that the torus link of type $(\alpha, \alpha)$ may be obtained from the two-bridged knot of type $(\alpha, 1)$ by this same construction.

13. Homotopy spheres as abelian coverings. Suppose $\pi: G(L) \rightarrow \delta\left(J_{n}\right)$ is the direct product $\pi_{1} \times \pi_{2}$ (see Definition 10.2), with notation as in Proposition 5.1 and the proof of Theorem 10.2. Then for each of $i=1,2$, the restriction of $\pi_{i}$ to a set of generators of $G\left(L_{i}\right)$ induces a transitive representation $\pi_{i}^{*}: G\left(L_{i}\right) \rightarrow \delta\left(J_{n_{1}}\right)$, and $n_{1} n_{2}=n_{0}$.

Proposition 13.1. If $\pi: G(L) \rightarrow \varsigma\left(J_{n}\right)$ is a direct product $\pi_{1} \times \pi_{2}$ of nontrivial representations $\pi_{1}$ and $\pi_{2}$, then the branched covering space $M_{\pi}(L)$ can be constructed as an abelian branched covering space of the 3-manifold $M_{\pi_{1}^{*}}\left(L_{1}\right)$. 
Proof. Note first that $M_{\pi_{1}^{*}}\left(L_{1}\right)=M_{\pi_{1}}(L)$; let $\tilde{M}_{1}$ denote that space, and let $\tilde{M}$ denote $M_{\pi}(L)$. We let $p_{1}: \tilde{M}_{1} \rightarrow S^{3}$ denote the covering projection, and $p_{1^{*}}$ the induced map of the fundamental group $G_{1}$ of $\tilde{M}_{1}-p_{1}^{-1}(L)$ into $G(L)$. Then $\tilde{\pi}_{2}=\pi_{2} p_{1^{*}}: G_{1} \rightarrow \mathcal{S}\left(J_{n_{2}}\right)$ is a transitive abelian representation, and $\tilde{M}$ is the branched covering space of $\tilde{M}_{1}$ associated with $\tilde{\pi}_{2}$ (branched along $p_{1}^{-1}(L)$ ). Indeed, since $\omega_{2}\left(t_{k}\right)=1$ for $k \in S_{1}, \tilde{\pi}_{2}$ induces a representation $\tilde{\pi}_{2}^{*}$ from the fundamental group of $\tilde{M}_{1}-p_{1}^{-1}\left(L_{2}\right)$ into $\delta\left(J_{n_{2}}\right)$, so $\tilde{M}$ is in fact the branched (along $\left.p_{1}^{-1}\left(L_{2}\right)\right)$ covering space of $\tilde{M}_{1}$ associated with $\tilde{\pi}_{2}^{*}$.

THEOREM 13.1. If the abelian branched covering space $M_{\pi}(L)$ is simply-connected, then it is $S^{3}$.

Proof. (The proof is by induction on $\mu$ and is based on a recent theorem (see Introduction) which proves the case $\mu=1$.) If any counterexample exists, let the covering $M_{\pi}(L)$ be a counterexample with minimum number $\mu$ of components; of course $\mu \geqslant 2$.

$M_{\pi}(L)$ is simply-connected and therefore a $\mathbf{Z}$-homology sphere; by Theorem 12.2(1), $\pi$ is the direct product $\pi_{1} \times \pi_{2} \times \cdots \times \pi_{\mu}$ of $\mu$ cyclic representations $\pi_{i}$ : $G\left(K_{i}\right) \rightarrow \varsigma\left(J_{r_{i}}\right)$.

Define $\pi_{0}=\pi_{1} \times \pi_{2} \times \cdots \times \pi_{\mu-1}$. Then $\pi=\pi_{0} \times \pi_{\mu}$; since $\mu$ is minimal, both $\pi_{0}$ and $\pi_{\mu}$ are nontrivial, and $n_{0}=n_{1} n_{2} \cdots n_{\mu-1}>1$.

Using Proposition 13.1 with all subscripts 1 and 2 changed to 0 and $\mu$, respectively, we see that $M_{\pi}(L)$ is a cyclic branched (along $p_{0}^{-1}\left(L_{\mu}\right)$ ) covering of $\tilde{M}_{0}$ associated with $\tilde{\pi}_{\mu}^{*}$, where $L_{0}=K_{1} \cup K_{2} \cup \cdots \cup K_{\mu-1}$, and $L_{\mu}=K_{\mu}$ is a knot, and $\tilde{M}_{0}=M_{\pi_{0}^{*}}\left(L_{0}\right)$ is a branched abelian covering of the $(\mu-1)$-component link $L_{0}$.

Since $M_{\pi}(L)$ is simply-connected, so is $\tilde{M}_{0}$; but then, since $\mu$ is minimal, $\tilde{M}_{0}$ must be $S^{3}$. Since each meridian of every component of $p_{0}^{-1}\left(L_{\mu}\right)$ is mapped by $\tilde{\pi}_{\mu}^{*}$ onto the same element of $\delta\left(J_{n_{\mu}}\right), M_{\pi}(L)$ is in fact a nontrivial strictly-cyclic covering of $S^{3}$ branched along $p_{0}^{-1}\left(L_{\mu}\right)$. If this last were a link with more than one component, Corollary 10.1.1 would give a contradiction since $n_{\mu}$ (which $>1$ ) would divide $\left|M_{\pi}(L)\right|($ which $=1)$; but if it were a knot (of one component) then $M_{\pi}(L)$ would be a simply-connected $n_{\mu}$-fold cyclic covering of $S^{3}$ branched over the knot $p_{0}^{-1}\left(L_{\mu}\right)$, which is impossible by the recent theorem (see Introduction) unless $p_{0}^{-1}\left(L_{\mu}\right)$ is unknotted and $M_{\pi}(L)$ is $S^{3}$. Thus no counterexample can exist.

14. Homology groups of unbranched covering spaces. In this section we state without proof a few results on the homology group of an unbranched abelian covering space of a link $L$ (see [15]).

With $U_{\pi}=U_{\pi}(L)$ and $M_{\pi}=M_{\pi}(L)$ as before, let $T\left(U_{\pi}\right)$ be the order of the torsion group of $H_{1}\left(U_{\pi}\right)$. Recall $\lambda_{i}=\left|\omega\left(t_{i}\right)\right|$.

Definition 14.1. If each component of $L$ is covered in $M_{\pi}$ by a single knot, we call the representation $\omega$ nondivisive.

THEOREM 14.1. If $\omega$ is nondivisive and $\left|H_{1}\left(M_{\pi}\right)\right|<\infty$, then

$$
T\left(U_{\pi}\right)=\frac{n}{\lambda_{1} \lambda_{2} \cdots \lambda_{\mu}} \cdot T\left(M_{\pi}\right) .
$$


COROLlaRY 14.1.1. If $M_{\pi}$ is a strictly-cyclic covering of a link $L$ with $\mu>2$ and $\left|H_{1}\left(M_{\pi}\right)\right|<\infty$, then

$$
T\left(U_{\pi}\right)=\prod_{j=1}^{n-1} \nabla_{L}\left(\eta^{j}\right),
$$

where $\eta$ is a primitive nth root of unity and $\nabla_{L}$ is the Hosokawa polynomial of $L$.

COROLlary 14.1.2. If $\mu=1$ and $\left|H_{1}\left(M_{\pi}\right)\right|<\infty$, then $T\left(M_{\pi}\right)=T\left(U_{\pi}\right)$.

The well-known Corollary 14.1.2 (which appears, for example, in [7]) implies that there is little additional information in the homology groups of $U_{\pi}$ in case $\mu=1$; the $U_{\pi}$ are manifolds with boundary, and thus more difficult to study than the $M_{\pi}$, so it is natural that they have been less studied heretofore. However, when the conditions of Theorem 14.1 are not satisfied, the structure of $U_{\pi}$ is often very rich, and may contain much additional information.

THEOREM 14.2. If $\omega$ is nondivisive and $\left|H_{1}\left(M_{\pi}\right)\right|<\infty$, then

$$
T\left(U_{\pi}\right)=\frac{1}{E(\omega)} \cdot \prod_{\zeta \in Z} \tilde{\Delta}_{L}\left(\zeta m_{1}, \zeta m_{2}, \ldots, \zeta m_{\mu}\right),
$$

where notation is as above and $E(\omega)$, the prolixity of the representation $\omega$, is an integer in the range $1 \leqslant E(\omega) \leqslant n^{\mu-2}$.

Since $T\left(U_{\pi}\right)$ must be an integer, this last theorem promises to impose new restrictions on the Alexander polynomials of links in $S^{3}$, and may shed light on the problem of characterizing those polynomials.

\section{REFERENCES}

1. J. W. Alexander, Topological invariants of knots and links, Trans. Amer. Math. Soc. 30 (1928), 275-306.

2. E. J. Brody, Topological classification of the lens spaces, Ann. of Math. (2) 71 (1960), 163-184.

3. G. Burde, On branched coverings of $S^{3}$, Canad. J. Math. 23 (1971), 84-89.

4. Verschlingungsinvarianten von Knoten und Verkettungen mit Zwei Brücken, Math. Z. 145 (1975), 235-242.

5. R. H. Fox, Free differential calculus. I, Ann. of Math. (2) 57 (1953), 547-560.

6. Free differential calculus. II, Ann. of Math. (2) 59 (1954), 196-210.

7. Free differential calculus. III, Ann. of Math. (2) 64 (1956), 407-419.

8. R. H. Fox, K. T. Chen and R. C. Lyndon, Free differential calculus. IV, Ann. of Math. (2) 68 (1958), $81-95$.

9. R. H. Fox, Free differential calculus. V, Ann. of Math. (2) 71 (1960), 408-422.

10. Covering spaces with singularities, Lefschetz Symposium, Princeton Math. Ser., vol. 12, 1957, pp. 243-257.

11. A quick trip through knot-theory, Topology of 3-Manifolds and Related Topics, Prentice-Hall, Englewood Cliffs, N. J., 1962.

12. A note on branched cyclic coverings of spheres, Rev. Mat. Hisp.-Amer. 32 (1972), 158-166.

13. R. H. Fox and G. Torres, Dual presentations of the group of a knot, Ann. of Math. (2) 59 (1954), 211-218.

13a. L. Goeritz, Die Betti'sche Zahlen der Zyklischen Überlagerungsräume der Knotenaussenräume, Amer. J. Math. 56 (1934), 194-198.

14. R. Hartley and K. Murasugi, Covering linkage invariants, Canad. J. Math. 19 (1977), 1312-1339.

15. _. Homology invariants, Canad. J. Math. 30 (1978), 655-670.

16. U. Hirsch and W. D. Neumann, On cyclic branched coverings of spheres, Math. Ann. 215 (1975), 289-291. 
17. F. Hosokawa and S. Kinoshita, On the homology group of the branched cyclic covering spaces of links, Osaka Math. J. 12 (1960), 331-355.

18. N. Jacobson, Lectures in abstract algebra. II, Van Nostrand, Princeton, N. J., 1952.

18a. P. Lancaster, Theory of matrices, Academic Press, New York, 1969.

19. J. P. Mayberry, Abelian branched coverings of knots, Ph.D. Thesis, Princeton, 1955, MIC 57-2160, University Microfilms, Ann Arbor, Michigan.

19a. J. M. Montesinos, 3-Variétés qui ne sont pas des revêtements cycliques ramifiés sur $S^{3}$, Proc. Amer. Math. Soc. 47 (1975), 495-500.

20. A. Plans, Contributions to the study of the homology groups of the cyclic ramified coverings corresponding to a knot, Rev. Real Acad. Cienc. Exact. Fis. Natur. Madrid 47 (1953), 161-193. (Spanish)

21. H. Seifert, Topologie dreidimensionaler gefaserter Räume, Acta Math. 60 (1933), 147-238.

22. __ Über das Geschlecht von Knoten, Math. Ann. 110 (1934), 571-592.

22.a. L. Siebenmann, On vanishing of the Rohlin invariant and non-finitely amphicheiral homology 3-spheres, Proc. Topology Sympos. (Siegen, 1979), Lecture Notes in Math., vol. 788, Springer-Verlag, Berlin and New York, 1980, pp. 172-222.

23. G. Torres, On the Alexander polynomial, Ann. of Math. (2) 57 (1953), 57-89.

24. O. Zariski, On the topology of algebroid singularities, Amer. J. Math. 54 (1932), 453-465.

Department of Mathematics, Brock University, St. Catharines (L2S 3A1), Ontario, Canada

Department of Mathematics, University of Toronto, Toronto (M5S 1A1), Ontario, Canada 